\title{
Alcune considerazioni in tema di infrastrutture: crisi, semplificazioni, finanziamenti e ruolo dello Stato-apparato ${ }^{1}$
}

\author{
GABRIELE TORELLI ${ }^{2}$ \\ ENRICO GUARNIERI ${ }^{3}$
}

\section{SINTESI}

Muovendo dal valore strategico-strumentale delle infrastrutture e dato conto dell'attuale deficit infrastrutturale in Italia, l'articolo analizza alcuni aspetti critici del settore. Il primo è quello della difficoltà di semplificare procedimenti complessi e costellati da interessi: sul punto, la via tracciata dall'ordinamento è quella del ritorno alla normativa "minimale" delle direttive europee. La seconda criticità è, invece, rinvenuta nelle difficoltà di reperire le risorse necessarie per promuovere gli investimenti, che hanno spesso consigliato

1 Il presente lavoro è frutto di approfondimenti e riflessioni comuni ${ }_{i}$ tuttavia, i paragrafi 1 e 2 sono stati scritti da Enrico Guarnieri, mentre i paragrafi 3, 4 e 5 da Gabriele Torelli.

2 Dottore di ricerca in scienze giuridiche Università di Bologna, Bologna, Italia. Assegnista di ricerca in Diritto amministrativo presso l'Università IUAV di Venezia, Venezia, Italia. Correo-e: gtorelli@iuav.it. Enlace ORCID: https://orcid.org/0000-0001-7048-0212

3 Dottore di ricerca in scienze giuridiche Università di Bologna, Bologna, Italia. Assegnista di ricerca in Diritto amministrativo presso l'Università di Bologna, Bologna, Italia. Correo-e: enrico.guarnieri3@unibo.it. Enlace ORCID: https://orcid.org/00000002-7599-9547. Fecha de recepción: 10 de febrero de 2021. Fecha de modificación: 10 de abril de 2021. Fecha de aceptación: 10 de mayo de 2021. Para citar el artículo: Torelli, Gabriele y GUARNieri, ENRICO, "Alcune considerazioni in tema di infrastrutture: crisi, semplificazioni, finanziamenti e ruolo dello stato-apparato", Revista digital de Derecho Administrativo, Universidad Externado de Colombia, n. ${ }^{\circ} 26,2021$, pp. 127-162. DOI: https://doi.org/10.18601/21452946.n26.05. 
all'amministrazione di definire dei PPP per beneficiare di capitali privati. Considerando che grazie al Next Generation EU l'Italia disporrà di ingenti finanziamenti europei, le risorse per la realizzazione delle opere pubbliche non mancheranno: per questo, lo studio intende valutare se il privato potrà rivestire ancora un ruolo nel progetto di rilancio infrastrutturale del Paese.

Parola chiave: infrastrutture, semplificazione, PPP, finanziamenti europei, potenziamento infrastrutturale

\section{Some Thoughts on Infrastructure: Crises, Simplification, Resources and the Role of the State}

\section{ABSTRACT}

This paper analyzes some critical aspects in view of the strategic role of infrastructures and considering its current deficit in Italy. The first aspect is the difficulty of simplifying complex and interest-ridden procedures, a point on which the Italian legal system's path is to return to the logic of the "minimum" regulatory frame in the European directives. The second critical aspect is to be found, on the contrary, in the difficulties of raising the necessary resources to promote investments, for which the Administration has turned to PPPs as an instrument to benefit from private capital. Thanks to the Next Generation EU, Italy will have significant European funding, so it is expected that there will be no shortage of resources for the construction of public works. As a result, this study aims to assess whether the private sector will still be able to play an important role in the country's infrastructure revitalization project.

Keywords: Infrastructure, Simplification, PPP, European Financing, Infrastructure Development 


\section{Algunas consideraciones relativas a la regulación de infraestructura: crisis, simplificaciones, financiamiento y papel del Estado}

\section{RESUMEN}

Partiendo del valor estratégico-instrumental de las infraestructuras y dado el actual déficit de las mismas en Italia, el artículo analiza algunos aspectos críticos en este sector. El primero consiste en la dificultad de simplificar procedimientos complejos y plagados de intereses; punto en el que el camino trazado por el ordenamiento jurídico italiano es regresar a la lógica de la regulación "mínima" de las directivas europeas. El segundo aspecto crítico se encuentra, por el contrario, en las dificultades para recabar los recursos necesarios destinados a promover las inversiones, para lo cual la Administración ha acudido a las APP como instrumento para beneficiarse del capital privado. Gracias a la Nueva Generación UE, Italia contará con una importante financiación europea, de manera que se espera que no haya escasez de recursos para la construcción de obras públicas. El presente estudio pretende por ende evaluar si el sector privado aún podrá desempeñar un papel importante en el proyecto de revitalización de la infraestructura del país.

Palabras clave: infraestructura, simplificación, APP, financiación europea, mejora de la infraestructura.

\section{CRISI INFRASTRUTTURALE IN ITALIA: UNA INTRODUZIONE}

In forza di una crisi governativo-istituzionale che ha doppiato quella pandemica, il problema del rilancio infrastrutturale del Sistema Paese è divenuto sempre più centrale nel dibattito pubblico italiano e, a seguito del recente insediamento del nuovo governo, ha assunto, anche per ragioni esogene (infra par. 3), una più marcata formalizzazione nell'agenda politica.

A spiegare l'importanza e l'urgenza del tema concorrono certamente ricorrenti ragioni di natura politica, di rappresentanza e, assieme, di soddisfazione di una parte della base elettorale di riferimento ${ }^{4}$. Eppure, la tentazione 
(che talvolta diviene tendenza) a risolvere l'allarme nel mero compiacimento di questa o quella compagine elettorale ${ }^{5}$ - banalizzando, costruttori edili, imprese, etc. - tradisce l'effettività e la gravità del problema.

L'infrastruttura, infatti, non è rilevante in sé e di per sé, così come - ed è questo il punto - il deficit infrastrutturale è altro dalla mera assenza o precarietà di beni immobili...la ragione? La ragione ha matrici essenzialmente teoriche e si fonda sull'autorevole affermazione secondo cui "gli approntamenti infrastrutturali sono opere pubbliche" ma "[n]on è vero l'inverso"6: si badi, non si tratta di un calembour, non fosse altro perché le implicazioni sono concretissime.

Individuati, infatti, nella dinamicità e nella strumentalità i tratti caratterizzanti e differenziali del concetto di infrastruttura - "[s]e il concetto di opera pubblica richiama l'idea statica del bene immobile, il concetto di infrastruttura, invece, implica quello più dinamico di bene strumentale" - la dottrina ha poi colto la vera essenza del problema: l'infrastruttura rileva "in ragione della funzione che la stessa può e deve svolgere: le infrastrutture, infatti, sono strumentali per lo più alla funzionalità di un luogo, all'approvvigionamento delle risorse, agli scambi tra le merci, alla mobilità e agli spostamenti delle persone ${ }^{\prime \prime 7}$.

Il retroterra culturale di queste affermazioni è peraltro considerevole, ancorché non necessariamente corrispondente sotto il profilo ideologico. Una sintetica ma importante traccia di quel background è fornita da Giacinto della Cananea in un saggio "inaugurale" della Rivista Munus, laddove le divergenti impostazioni di Adam Smith e di John Maynard Keynes sono fatte convergere, per l'appunto, attorno all'importanza e alla centralità del ruolo dello Stato nell'approntamento delle infrastrutture utili al benessere della società ${ }^{8}$. Ma in quella sede a trovare spazio è anche l'idea, qui rilevantissima, secondo cui il valore degli investimenti pubblici dipende "dalla misura in cui le infrastrutture e le reti [...] recano un apporto effettivo al progresso tecnico e civile": ritorna, così, attraverso il tema dell'efficienza della spesa (infra par. 3), la concezione funzional-strumentale delle infrastrutture.

Napoli: Editoriale Scientifica, 2013, p. 8, "[p]er gli organi politici, poi, i contratti dell'amministrazione hanno talora purtroppo costituito un essenziale - ma distorto strumento di gestione del consenso".

5 Michele Serra, "Grandi opere. Gli ostinati del bluff", in La Repubblica, 8 agosto 2006.

6 Massimo Severo Giannini, Diritto pubblico dell'economia, Bologna: il Mulino, 1993, p. 57.

7 GiUSEPPE PIPERATA, "Infrastrutture e crescita economica: alla ricerca di nuovi modelli", in Munus, n. 2, IV, 2013. Sul punto, si v. anche Alessandro Crosetti, "Dalle opere pubbliche alle infrastrutture: profili evolutivi", in La disciplina delle opere pubbliche, a cura di Alessandro Crosetti, Rimini: Maggioli, 2007, p. 54.

8 Giacinto Della Cananea, "Per un nuovo assetto delle reti di servizi pubblici", in Munus, n. ${ }^{\circ} 1,2011$, pp. 105-106. 
Parrà quindi chiaro che parlare di infrastrutture - siano esse strategiche (in senso giuridico) ${ }^{9} \mathrm{o}$ meno - significa discorrere di sostegno all'economia, di competitività del Paese, di libertà e di diritti costituzionalmente tutelati (movimento, salute, educazione, etc.), nonché, con una certa corrispondenza, di servizi pubblici ${ }^{10}$ - della loro qualità ${ }^{11}$, della continuità dell'erogazione e della economicità della gestione ${ }^{12}-\mathrm{e}$, in definitiva, di coesione sociale-economica ${ }^{13}$; correlativamente, affrontare il problema infrastrutturale significa trattare di crisi: crisi al plurale, queste potendo investire ciascuno degli aspetti ora menzionati.

9 Artt. 200-203, d.lgs. n. 50/2016.

10 Ponendo alla base del ragionamento la ricordata distinzione tra opera pubblica ed infrastruttura, RUGGIERO DIPACE, "Le reti di trasporto fra disciplina europea e nazionale per la realizzazione di una mobilità sostenibile", in Infrastrutture di trasporto e sistemi di regolazione e gestione. Coesione, sostenibilità e finanziamenti, a cura di Giovanna Colombini, Marina D'Orsogna, Loredana Giani e Aristide Police, Napoli: Editoriale Scientifica, 2019, p. 31, ricorda, infatti, che "le infrastrutture sono funzionali alla erogazione di servizi pubblici".

11 Cfr. Marco Dugato, "La crisi del concetto di servizio pubblico locale tra apparenza e realtà", in Diritto Amministrativo, n. ${ }^{\circ} 3,2020$, p. 518, il quale, con affermazioni sì riferite ad un preciso periodo storico (la seconda metà degli anni '80) ma qui generalizzabili, afferma che la "pressante domanda di miglioramento della qualità dei servizi" implica quale "tema nodale" quello della "qualità delle infrastrutture", che, in particolare per i servizi "a rete", costituisce "l'elemento cardine del miglioramento".

12 Infrastrutture scadenti o mancati possono implicare, infatti, interruzioni del servizio o un aumento dei costi di gestione (si pensi solo, per fare un esempio, alla necessità di mettere in strada più mezzi pubblici di trasporto per compensare i ritardi nelle corse). Sul principio di economicità quale criterio "normale" di gestione di servizi pubblici continuativi e qualitativi, cfr. GIUSEPPE CAIA, "Criterio di economicità e servizi pubblici locali nella prospettiva della XVIII legislatura repubblicana", in Sabino Cassese et al., Diritto amministrativo e società civile, I, Studi introduttivi, Bologna: Bononia University Press, 2018, pp. 327 y ss.: "i servizi pubblici si devono intendere retti dal criterio di economicità quale conseguenza necessaria dell'esigenza di espletarli con continuità. Se il servizio pubblico è ritenuto necessario per la collettività ed è stato di conseguenza organizzato (istituito), la relativa gestione non può essere occasionale o transeunte, ma deve essere destinata a durare. La garanzia concreta della predetta continuità di esercizio può essere data solo dalla economicità gestionale, quale fattore di equilibrio idoneo a scongiurare o comunque contrastare interruzioni o scadimento di qualità" (343). Nello stesso senso, cfr. GIUSEPPE CAIA, "Il trasporto pubblico locale come paradigma del servizio pubblico (disciplina attuale ed esigenze di riordino)", in Rivista AIC, n. ${ }^{\circ} 3,2018$, p. 335 .

13 Cfr. MARIO SEBASTIANI, "Le infrastrutture di trasporto: programmazione, concorrenza, interesse pubblico", in I nodi delle reti. Infrastrutture, mercato e interesse pubblico, a cura di Paola Manacorda, Firenze: Passigli Editori, 2010, p. 47, secondo il quale "[i]ndipendentemente dai settori [...] le infrastrutture costituiscono fattore di sviluppo economico e condizione essenziale per la coesione territoriale, sociale, economica e politica di una collettività". 
Sotto questo profilo, uno degli esempi più recenti e maggiormente significativi della polifunzionalità delle infrastrutture è portato dalla disciplina delle reti TEN T, ovvero della rete transeuropea dei trasporti. Particolarmente emblematico, è il considerando n. 2, reg. Ule n. 1315/2013, laddove, intercettata la connessione con i goals della strategia Europa 2020, si afferma che la pianificazione, lo sviluppo e il funzionamento delle reti TEN T contribuiscono al raggiungimento di fondamentali obiettivi dell'Unione, quali "il buon funzionamento del mercato interno e il rafforzamento della coesione economica, sociale e territoriale". Ma non è tutto: l'implementazione della rete transeuropea dei trasporti persegue "tra gli altri, anche gli obiettivi specifici di consentire la mobilità senza ostacoli, sicura e sostenibile delle persone e delle merci e di permettere l'accessibilità e la connettività a tutte le regioni dell'Unione, contribuendo all'ulteriore crescita economica e alla competitività in una prospettiva globale ${ }^{\prime \prime 14}$. Inutile dirsi che un deficit infrastrutturale in questo settore comporterebbe molteplici e rilevantissime ripercussioni in negativo su ciascuno di quegli obiettivi (i.e. interessi), tra cui quello, sopra imprecisamente obliterato, della sostenibilità ${ }^{15}$.

Compresa, quindi, la fisiologia, ovvero la ragione che, per dirla con Giannini, fa dell'approntamento delle infrastrutture una delle "più antiche attività di disciplina dell'economia svolte dai pubblici poteri"16, rimane da considerare il profilo patologico della vicenda.

L'antichissima attività di approntamento delle infrastrutture, scriveva sempre Giannini negli anni Novanta, "non è mai venuta meno lungo il corso dei secoli; oggi non solo permane, ma ha assunto dimensioni grandissime, sia quantitative che qualitative" ${ }^{117}$.

L'oggi odierno è molto diverso. Da più parti si lamenta un problema infrastrutturale alquanto diffuso ${ }^{18}$, seppur differenziato tra il Nord e il Sud

14 Per una esaustiva analisi della disciplina recata dal reg. Ue n. 1315/2013, si v. RuGGIERO DIPACE, "Le reti di trasporto fra disciplina europea e nazionale per la realizzazione di una mobilità sostenibile", pp. 31 e ss.

15 Tema rispetto al quale si saldano le dinamiche (in atto) relative al trasporto pubblico locale e alle "sue" infrastrutture. In merito, ed in particolare, con riferimento alla "(ri) configurazione del trasporto pubblico quale elemento fondativo della sostenibilità della trama territoriale e insediativa, anche sotto il profilo dell'equità sociale, e di cui deve essere pertanto promosso il potenziamento sia sul piano infrastrutturale sia per quanto concerne la qualità e la quantità dei servizi erogati all'utenza", cfr. TOMMASO BONETTI, "Il trasporto pubblico locale nel prisma della mobilità sostenibile", in Diritto amministrativo, v. 3, 2020, pp. 563 e ss. spec. 583.

16 Massimo Severo Giannini, Diritto pubblico dell'economia, p. 53.

17 Ibid. (carattere c.vo. aggiunto da chi scrive).

18 Di recente, cfr. DARIO IMMORDINO, "Recovery plan? Sostegno allo sviluppo ed equilibrio finanziario con il 'tesoro' dei lavori pubblici", in Appalti e contrati, n. ${ }^{\circ}$ 7-8, 2020, pp. 15 e ss. 
del Paese $\mathrm{e}^{19}{ }_{;}$ma questa diversità giace, per l'appunto, sul piano quantitativo e qualitativo, non investendo invece il profilo, per così dire, modale: una recente analisi dell'evoluzione delle politiche del settore ha infatti reso "evidente" che l'approntamento delle infrastrutture costituisce ancora "una "attività di disciplina dell'economia" di stretta attualità", per quanto "connotata da una innovativa dimensione sovranazionale e da un multiforme intervento dei pubblici poteri" 20 .

Dati alla mano, emergono almeno due aspetti degni di nota.

Il primo riguarda la dotazione e l'adeguatezza infrastrutturale. Un recente report del World Economic Forum (2020) colloca l'Italia al $18^{\circ}$ posto su 141 Stati considerati, con un miglioramento di nove posizioni rispetto all'indagine del 2017-2018, ma con un marcato distacco dai maggiori paesi europei (Paesi Bassi 2i, Spagna 7 ${ }^{\mathrm{a}}$, Germania ${ }^{\mathrm{a}}$, Francia $9^{\mathrm{a}}{ }^{21}$.

\begin{tabular}{|c|c|c|c|c|}
\hline Index Component & Value & Score * & Rank/141 & Best Performer \\
\hline 虫 2nd pillar: Infrastructure $0-100$ & $\cdot$ & $84.1 \uparrow$ & 18 & Singapore \\
\hline Transport infrastructure $0-100$ & - & $73.2 \uparrow$ & 17 & Singapore \\
\hline 2.01 Road connectivity 0-100 (best) & 85.9 & $85.9 \uparrow$ & 38 & Multiple (3) \\
\hline 2.02 Quality of road infrastructure $1-7$ (best) & 4.4 & $56.8 \uparrow$ & 53 & Singapore \\
\hline 2.03 Railroad density km/1,000 km[1]2 & 57.1 & $100.0=$ & 16 & Multiple (24) \\
\hline 2.04 Efficiency of train services $1-7$ (best) & 4.1 & $52.0 \uparrow$ & 35 & Japan \\
\hline 2.05 Airport connectivity score & $616,580.8$ & $97.1=$ & 11 & Multiple (8) \\
\hline 2.06 Efficiency of air transport services 1-7 (best) & 4.9 & $65.4 \uparrow$ & 55 & Singapore \\
\hline 2.07 Liner shipping connectivity 0-100 (best) & 67.2 & $67.2 \uparrow$ & 20 & Multiple (5) \\
\hline 2.08 Efficiency of seaport services $1-7$ (best) & 4.7 & $61.1 \uparrow$ & 46 & Singapore \\
\hline Utility infrastructure $0-100$ & - & $95.0 \uparrow$ & 31 & Iceland \\
\hline 2.09 Electricity access \% of population & 100.0 & $100.0=$ & 2 & Multiple (67) \\
\hline 2.10 Electricity supply quality \% of output & 5.7 & $98.2 \uparrow$ & 30 & Multiple (10) \\
\hline 2.11 Exposure to unsafe drinking water \% of population & 0.5 & $100.0=$ & 21 & Multiple (28) \\
\hline 2.12 Reliability of water supply $1-7$ (best) & 5.9 & $81.8 \uparrow$ & 40 & Iceland \\
\hline
\end{tabular}

Fonte: World Economic Forum, The Global Competitiveness Report 2019.

La stima conferma il giudizio tendenzialmente negativo formulato in uno studio del 2019 promosso dalla Banca d'Italia, ove, seppur con le dovute cautele (motivate dalla mancanza di uniformità/condivisione delle metodologie

19 Cfr. AsSociaZIONE PER lo SVILUPPO DEll'INdustria nEl MEZZOGIORnO (SVIMEZ), I divari infrastrutturali e la spesa per opere pubbliche: la mancata attuazione della perequazione infrastrutturale, 2019. Per una approfondita analisi della "questione del Mezzogiorno", cfr. SVIMEZ, Il Mezzogiorno nella storia economica d'Italia. Una questione aperta, Roma, 2017; SABINO CASSESE, "Dallo sviluppo alla coesione. Storia e disciplina vigente dell'intervento pubblico per le aree insufficientemente sviluppate", in Rivista Trimestrale di diritto pubblico, n. ${ }^{\circ} 2,2018$, pp. 579 e ss.

20 Susanna SCREPANTI, "Le politiche infrastrutturali", in La nuova costituzione economica, a cura di Sabino Cassese, Bari-Roma: Laterza, 2021, p. 37.

21 World Economic Forum, The Global Competitiveness Report 2019, Swizerland, 2019, pp. 41 e ss. 
di calcolo e dalla soggettività delle valutazioni dei soggetti intervistati), si è affermato che " 1 'Italia si trova in una posizione non favorevole in termini di quantità e qualità dello stock di infrastrutture, e che la situazione è peggiorata negli ultimi anni relativamente agli altri paesi"22.

Il secondo aspetto attiene, invece, alle fonti di finanziamento e alle procedure di spesa: il mito è stato sfatato, le risorse ci sono ${ }^{23}-$ ad esempio, secondo i dati forniti dal Servizio Studi della Camera dei Deputati, la copertura delle opere strategiche programmate ammonta, rispettivamente per quelle prioritarie e non prioritarie, al $73 \%$ e all' $81 \%$ dei costi previsti ${ }^{24}$ - semmai il problema è spenderle (infra par. 3). Su questo fronte, gli sviluppi della doppia impasse "blocco della spesa" e "blocco delle opere" mostrano tendenze in parte positive, benché ancora non del tutto soddisfacenti. Da un lato, il superamento del pareggio di bilancio e lo sblocco degli avanzi di amministrazione ha consentito una decisa ripresa delle spese in conto capitale dei comuni italiani, che per l'anno 2019 segnano un incremento del $16 \%$; però, il gap da recuperare è molto, tenuto conto che il decennio che ha seguito la crisi economico-finanziaria globale del 2008 ha comportato una contrazione della spesa per investimenti maggiore del $54 \%$ : come osservato dall'Associazione nazionale costruttori edili (ANCE), "[a]nche confermando una crescita del $16 \%$ nel 2019, gli investimenti in conto capitale dei comuni sarebbero comunque inferiori di circa il $47 \%$ rispetto al livello conseguito nel 2008"25. Dall'altro lato, cioè sul fronte del "blocco delle opere", i segnali mostrano una leggera riduzione delle opere incompiute. Elaborando i dati forniti dal Sistema Informativo Monitoraggio Opere Incompiute (SIMOI), ad oggi risultano circa 420 opere incompiute, con un decremento significativo rispetto alla rilevazione relativa all'anno 2018 (più di 100 unità ${ }^{26}$, ma i dati sono estremamente variabili, vuoi per gli aggiornamenti regionali vuoi per

22 Fabio BUSETTI et al., "Capitale e investimenti pubblici in Italia: effetti macroeconomici, misurazione e debolezze regolamentari", in Banca d'Italia-Eurosistema. Questioni di Economia e Finanza (Occasional Papers), n. ${ }^{\circ}$ 520, ottobre 2019, pp. 13 e ss. Sul punto, si v. anche il contributo del Governatore della Banca d'Italia IGNAZIO VISCO, "Investimenti pubblici per lo sviluppo dell'economia", in Sviluppo economico, vincoli finanziari e qualità dei servizi: strumenti e garanzie. Atti del LXIV Convegno di studi di scienza dell'amministrazione promosso da Provincia di Lecco, Corte dei conti, Varenna-Villa Monastero, 20-21-22 settembre 2018, Milano: Giuffrè, 2019, pp. 271 e ss.

23 Susanna SCREPANTI, "Le politiche infrastrutturali", pp. 40 e ss.

24 Servizio Studi Della Camera Dei DePUTATI, Infrastrutture strategiche e prioritarie. Programmazione e realizzazione. Rapporto 2020 in collaborazione con l'Autorità nazionale anticorruzione, Roma, 2020, p. 13.

25 AnCe-Direzione AfFari ECOnOmici e Centro StUdi, Osservatorio congiunturale sull' industria delle costruzioni, Roma, 2020, pp. 91-92

26 Cfr. i dati forniti dal dossier della FONDAZIONE ERGO, L'Italia in-compiuta un'analisi economica delle opere pubbliche. I quaderni di approfondimento, n. ${ }^{\circ} 6,2019$, pp. 5-8. 
l'uso di piattaforme diverse rispetto al SIMOI): il trend è quindi positivo, ma gli esiti non sono ancora soddisfacenti, soprattutto se si pensa che secondo una stima effettuata dall'ANCE per l'anno 2018 "[dal]la realizzazione delle 574 infrastrutture bloccate su tutto il territorio nazionale oltre al miglioramento della competitività del Paese e della qualità della vita di cittadini e imprese del territorio, deriverebbe una ricaduta positiva sull'economia pari a 137 miliardi di euro e una spinta all'occupazione con la creazione di oltre 600 mila posti di lavoro" 27.

Come rilevato da più parti, peraltro, sul sistema pesano i rallentamenti degli iter procedurali: "il ritardo del nostro Paese sugli investimenti pubblici riguard[a] non tanto il volume di risorse finanziarie disponibili, quanto l'effettiva realizzazione di infrastrutture ${ }^{\prime 28}$. Dai dati elaborati dalla Agenzia per la Coesione Territoriale, infatti, il tempo medio di realizzazione di un'opera ammonta a circa 4 anni e 5 mesi (si va dai 3 anni per i lavori di importo inferiore a 100.000 euro ai 15,7 anni per i progetti con valore superiore a 100 milioni di euro). A pesare maggiormente sono i tempi "di attraversamento" (c.d. tempi della burocrazia) - intervallo temporale tra il termine di una fase e l'inizio dell'altra - che nei tre stadi della progettazione superano sempre la durata della fase "effettiva" (basti solo pensare che i tempi di attraversamento costituiscono il $68,7 \%$ di tutta la durata della fase di progettazione di fattibilità tecnico-economica) ${ }^{29}$.

Il quadro critico così sommariamente delineato pone l'interprete dinanzi alla eterogenesi delle soluzioni. Una fra tutte, quella del coordinamento infrastrutturale tra i livelli di governo nazionale e territoriale, meriterebbe un capitolo a parte. Marco Cammelli ricorda opportunamente come il "territorio [sia] un "pieno" di livelli sovrapposti di regolazione, di relazioni dirette con reti ed attori globali, di cooperazioni verticali e orizzontali, di politiche generali e di settore ${ }^{1130}$; ebbene, anche in ragione del fallimento della riforma costituzionale del 2016, in quel "pieno" il quesito sul "chi fa che $\operatorname{cosa}^{\prime \prime 31}$ rimane ora come allora insoluto. A tal riguardo, sorprende leggere che "[n]ell'area delle infrastrutture sta un nodo irrisolto. L'esperienza degli

27 AnCE-Direzione Affari ECONOMiCi e Centro StUdi, Osservatorio congiunturale sull' industria delle costruzioni, p. 130.

28 Fabio Busetti et al., "Capitale e investimenti pubblici in Italia", p. 20.

29 Agenzia Per la Coesione Territoriale, Rapporto sui tempi di attuazione delle opere pubbliche, Roma, 2018, pp. 15-19. In dottrina, sul tema, cfr. SuSANA SCREPANTI, "I tempi delle opere pubbliche e la relatività del tempo nel diritto amministrativo", in Riv. trim. dir. pubbl., n. ${ }^{\circ}$ 4, 2019, pp. 1189 e ss.

30 Marco CammelLI, "Istituzioni pubbliche e sviluppo economico locale", in Ist. fed., n. ${ }^{\circ} 2$ 2009, p. 223.

31 GiUSEPPE PIPERATA, "Infrastrutture e crescita economica: alla ricerca di nuovi modelli", p. IV. 
ultimi anni, dopo la riforma del Titolo V della Costituzione, mostra come il processo decisionale condiviso fra Stato e Regioni sia faticoso e spesso inefficace. Nell'interesse generale, occorre riflettere sui casi in cui è opportuno, trascorso un tempo definito, svincolare l'azione del governo centrale dall'obbligo di assenso degli enti regionali e locali interessati. Dare voce alle esigenze locali deve essere possibile senza bloccare sine die la realizzazione di opere necessarie alla modernizzazione del Paese". Ma lo stupore non è dovuto al contenuto dell'affermazione, quanto piuttosto alla fonte, perché quelle sono le parole con cui nel 2007 l'allora Governatore della Banca d'Italia Mario Draghi salutava il tema infrastrutturale ${ }^{32}$. Oggi, per tornare a quanto si diceva in apertura del contributo, Mario Draghi è Presidente del Consiglio dei Ministri: la speranza è, quindi, che quel quesito trovi pronta risposta.

Quanto alle restanti soluzioni, esse paiono giacere sul piano della semplificazione e su quello dell'incremento delle fonti di finanziamento. Due livelli, questi, fortemente interconnessi, tenuto conto che, per quanto si è potuto evincere dai dati passati in rassegna, il contributo offerto dall'aumento delle risorse dipende primariamente dall'efficacia dei procedimenti di spesa, ovvero dalla effettiva e tempestiva ultimazione dei lavori. I movimenti ordinamentali sul punto sono peraltro in atto: in risposta alla crisi pandemica, infatti, sono state dapprima avviate importanti politiche di semplificazione (infra par. 2), oggi affiancate da significativi interventi di finanziamento pubblico in corso di definizione (infra par. 3).

\section{CONTRATTI PUBBLICI E LA DIFFICOLTÀ DI SEMPLIFICARE LA COMPLESSITÀ. IL RILANCIO INFRASTRUTTURALE PASSA PER IL RITORNO ALLA DISCIPLINA EUROPEA}

È passato ben più di un secolo da quando la normativa sui contratti pubblici è stata per la prima volta disciplinata nell'Italia unita (1. 20 marzo 1865, n. 2248 , All. F.), e sembra un paradosso come ancora oggi sia diffusa una forte insoddisfazione nei confronti della regolazione.

Non ha certo contribuito alla chiarezza della materia l'avalanche $e^{33}$ normativa che ha investito il settore, tenuto conto che il cambiamento, se frenetico,

32 Mario Draghi, Considerazioni finali - Banca d'Italia, Assemblea Ordinaria dei Partecipanti anno 2006, Roma, 31 maggio 2007, p. 12.

33 Si richiama l'espressione "the avalanche of administrative law" di David Roberts, Victorian Origins of the British Welfare State, New Heaven: Yale University Press, 1960, p. 300, poi recuperata da SABINO CASSESE, Il diritto amministrativo: storia e prospettive, Milano: Giuffrè, 2010, spec. p. 33. 
comporta instabilità o, quantomeno, incertezza applicativa ${ }^{34}$. La materia, infatti, ha conosciuto sì evoluzioni, per così dire, stagionali (dall'imprinting contabilistico a quello pro-concorrenziale, per poi giungere all'attuale sistemazione strategica di promozione delle secondary policies) ${ }^{35}$, ma, nell'ambito di ogni stagione normativa, la regolazione è stata poi oggetto di continui interventi legislativi in veste "correttiva", efficacemente compendiati nella formula "stagione dei correttivi"36.

Ciò che più sorprende, e che a maggior ragione può sorprendere il lettore estero, è la ricorrenza con cui il tema della semplificazione si è mostrato nel corso dei decenni. Ed ha ragione Aldo Sandulli quando scrive che, in questo settore, è "[troppo] facile usare il termine semplificazioni"37.

Semplificazione è termine anfibio ${ }^{38}$.

Vi è anzitutto la semplificazione organizzativa, quella procedimentale e quella provvedimentale ${ }^{39}$ : già sotto questo profilo l'individuazione di ciò che si può e di ciò che si deve semplificare non è compito agile. Qualcosa, certo, è stato fatto: inter alia, la riduzione delle stazioni appaltanti ${ }^{40}$ (semplificazione organizzativa), la contrazione dei termini delle procedure di gara e l'informatizzazione delle stesse (semplificazione procedimentale), così come

34 Cfr. Maria AlesSandRa SANDulli, "'Principi e regole dell'azione amministrativa': riflessioni sul rapporto tra diritto scritto e realtà giurisprudenziale", in federalismi.it, n. ${ }^{\circ} 23$, 2017, a cui segue MARIA ALESSANDRA SANDULLI, "Ancora sui rischi dell'incertezza delle regole (sostanziali e processuali) e dei ruoli dei poteri pubblici. Postilla a 'Principi e regole dell'azione amministrativa. Riflessioni sul rapporto tra diritto scritto e realtà giurisprudenziale'", in federalismi.it, n. ${ }^{\circ} 11,2018$.

35 Per un'ampia e approfondita lettura delle stagioni normative attraverso i principi $c f r$. Mario Pilade Chiti, "Principi", in Trattato sui contratti pubblici, I, diretto Maria Alessandra Sandulli e Rosanna de Nictolis, Milano: Giuffrè, 2019, pp. 285 e ss., nonché, nello stesso volume, AlBerTo MASSERA, "Principi procedimentali", in Trattato sui contratti pubblici, I, diretto Maria Alessandra Sandulli e Rosanna De Nictolis, Milano: Giuffrè, 2019, pp. 332 e ss.

36 Gianfrancesco FidOne, "Il nuovo codice dei contratti pubblici", in Commentario al codice dei contratti pubblici, a cura di Marcello Clarich, Torino: Giappichelli, 2019, p. 18.

37 Così Aldo SAndulli nell'Intervista di V. Conte pubblicata su La Repubblica, 12 febbraio 2021.

38 Cfr. Rosario Ferrara, Introduzione al diritto amministrativo, Roma-Bari: Laterza, 2014 p. 156, secondo il quale la semplificazione è fenomeno "plurimo e pluristrutturato" ed è espressione "marcatamente polisemica, e dunque apprezzabile sul piano meramente descrittivo".

39 Sul punto, si v. LuISA TORCHIA, "Tendenze recenti della semplificazione amministrativa", in Diritto amministrativo, n. ${ }^{\circ} 3-4,1998$, pp. 385 e ss.

40 Cfr. MARCO MACCHIA, "La qualificazione delle amministrazioni appaltanti", in Giornale di Diritto amministrativo, Fasc.1, 2017, pp. 50 e ss., e Bernardo Giorgio MatTarella, "La centralizzazione delle committenze", in Giornale di diritto amministrativo, n. ${ }^{\circ}$ 5, 2016, pp. 613 e ss. 
l'applicazione dei meccanismi acceleratori in relazione ai pareri del Consiglio superiore dei lavori pubblici ${ }^{41}$ e agli atti concertativi della conferenza di servizi sui progetti di fattibilità ${ }^{42}$ (semplificazione provvedimentale).

Ma la semplificazione reclama anche flessibilità: esemplificando, per facilitare il proprio studio e assieme per migliorare l'esito della commessa, una amministrazione che "sa di non sapere abbastanza" ${ }^{143}$ deve potersi rivolgere all'esterno per acquisire una consulenza, e ciò, oggi più di ieri, è possibile fare tramite le consultazioni preliminari di mercato (art. 66, d.lgs. n. 50/2016). Sennonché, rimossi i vincoli di matrice contabilistica ed espansa l'area della flessibilità ${ }^{44}$ (si pensi alla formale estensione del dialogo, oggi rilevante tanto nel pre-gara quanto nel corso delle procedure competitive ${ }^{45}$ ), si è poi ritratta nuovamente quella della semplificazione.

Per spiegarne la ragione è sufficiente fare riferimento a due dati ordinamentali.

Da un lato, il settore è imperversato da interessi, e si è lontani dall'interesse primario di Giannini. Nella materia dei contratti si impongono tanti interessi primari (in senso generico o in senso normativo ${ }^{46}$, poco importa) di segno positivo (massima partecipazione, sostenibilità, occupazione) o negativo (anti-corruzione): ciò che conta è (rectius: si direbbe essere) l'interesse pubblico in concreto (la sintesi degli interessi pubblici, tenendo conto di quelli privati $\left.{ }^{47}\right)$. Dall'altro lato, però e al contempo, il settore non è ancora pienamente improntato all'"amministrazione di risultato", o, meglio, lo è nella fase di gara - va in questo senso la conferma del ristretto ambito applicativo dell'immediata impugnazione dei bandi di gara ${ }^{48}$ e, per converso, il ripen-

41 Rosanna De Nictolis, "Le novità sui contratti pubblici recate dal D.L. n. 32/2019 'sblocca cantieri'", in Urbanistica e. appalti., n. ${ }^{\circ} 4,2019$, p. 461.

42 Per uno sguardo d'insieme, tra riforma della conferenza dei servizi nell'ambito della legge generale sul procedimento e funzionamento nel settore dei contratti pubblici, si v. RugGiero Dipace, "Procedure di approvazione dei progetti e conferenza di servi$\mathrm{zi}^{\prime \prime}$, in Trattato sui contratti pubblici, I, a cura di Maria Alessandra Sandulli e Rosanna De Nictolis, tomo IV, Milano: Giuffrè, 2019, p. 1353.

43 Emanuele Boscolo, "Consultazioni preliminari di mercato e possibili limitazioni alla concorrenza", in Giur. it., n. ${ }^{\circ}$ 1, 2020, p. 170.

44 Cfr. GiAn DOMENICO COMPORTI, "La flessibilità nelle negoziazioni pubbliche: questa sconosciuta", in Dir. soc., n. ${ }^{\circ} 2,2017$, pp. 177 e ss.

45 Fortunato Gambardella, Le regole del dialogo e la nuova disciplina dell'evidenza pubblica, Torino: Giappichelli, 2016, passim.

46 Si v. FRANCESCA TRIMARCHI BANFI, "I rapporti contrattuali della pubblica amministrazione", in Dir. pubbl., 1998, p. 37.

47 Cfr. Franco Gaetano Scoca, "Il coordinamento e la comparazione degli interessi nel procedimento amministrativo", in Convivenza nella libertà. Scritti in onore di Giuseppe Abbamonte, II, Napoli: Jovene, 1999, pp. 1273-1275.

Cons. St., ad. plen., 26 aprile 2018, n. 4. 
samento sul c.d. rito super accelerato ${ }^{49}-$ ma non lo è nella prospettiva della vicenda contrattuale unitariamente intesa ${ }^{50}$.

Dunque, se è l'aggiudicazione il risultato che conta (l'interesse della dir. 2014/24/Ue per la fase esecutiva rileva anche in quest'ottica ${ }^{51}$ ), allora la flessibilità da strumento utile per il raggiungimento dell'interesse pubblico concreto diviene un viatico paradigmatico di complicazioni. Per fare un esempio abbastanza calzante, basti pensare proprio alla disciplina delle consultazioni preliminari dei partecipanti al mercato, che espone l'amministrazione al problema, affatto irrilevante, di strutturare il quesito e la relativa procedura in modo tale da ottenere un'utilitas dal confronto, ma senza precludere all'operatore economico la possibilità di partecipare alla successiva ed eventuale gara ${ }^{52}$. D'altronde, la relazione tra la flessibilità e la concorrenza è, per certi versi, antagonistica. Vero è che la concorrenza, così come la matrice privatistica, nei modelli flessibili può trovare una minore mortificazione: si stempera il "matrimonio per corrispondenza"53, si sviluppa il momento della negoziazione e lo si valorizza applicandogli lo statuto della responsabilità precontrattuale ${ }^{54}$. Però, la flessibilità (quantomeno dal punto di vista del privato e, solo in parte, dell'ordinamento) è anche fonte di possibili storture concorrenziali: non è un caso che proprio laddove vi è flessibilità, tanto la dir. 2014/24/Ue quanto il codice domestico si premurano di predisporre accorgimenti (si badi, più) utili a salvaguardare la concorrenza (come bene ${ }^{55}$, che come strumento utile per il risultato).

49 Fino all'abrogazione disposta dal c.d. d.1. "sblocca cantieri" (d.1. 18 aprile 2019, n. 32, convertito con modificazioni dalla 1. 14 giugno 2019, n. 55), l'art. 120, comma 2-bis, d.lgs. 2 luglio 2010, n. 104, prevedeva l'onere di immediata impugnazione delle ammissioni ed esclusioni all'esito della valutazione dei requisiti generali e speciali: come noto, la previsione, disponendo, per il caso di omessa impugnazione, la preclusione "della facoltà di far valere l'illegittimità derivata dei successivi atti delle procedure di affidamento, anche con ricorso incidentale", aveva comportato un aumento esponenziale dell'anticipazione del contenzioso.

50 Per C. conti, Audizione della corte dei conti nell'ambito dell' "attività conoscitiva sull'applicazione del d.lgs. 18 aprile 2016, n. 50 (codice dei contratti pubblici)", Senato della Repubblica, 2019, 19, "[i]l Codice è prevalentemente incentrato sulla fase di selezione del contraente mentre appare limitata la riflessione in ordine sia alla valutazione ex ante delle finalità cui mira l'opera pubblica ed i bisogni che si intendono soddisfare attraverso di essa, sia al controllo concomitante, sia al controllo ex post dell'avvenuto perseguimento di tali obiettivi".

51 Utili considerazioni possono essere tratte da ANNALAURA GianNELLI, "Contratti pubblici: stabilità del rapporto e interessi pubblici", in Diritto economia, n. ${ }^{\circ} 1,2016$, pp. 127 e ss.

52 Cfr. le linee guida n. 14, recanti "Indicazioni sulle consultazioni preliminari di mercato", adottate dall'ANAC nel marzo 2019.

53 Giulio Napolitano, La logica del diritto amministrativo, Bologna: il Mulino, 2020, p. 316.

54 Cons. St., ad. plen., 4 maggio 2018, n. 5.

55 Per considerazioni critiche sulla concorrenza come bene cfr. FRANCESCA TRIMARCHI BANFI, "Il 'principio' di concorrenza: proprietà e fondamento", in Diritto amministrativo, 
Ebbene, si è detto sopra del paradosso secondo il quale una normativa tanto risalente sia ancora esposta a incessanti critiche. Ma si comprenderà ora che il paradosso è solo apparente: è il portato della complessità, ovvero della molteplicità degli interessi pubblici e privati che convergono sulla materia. Vero è che l'eterogenesi dei fini tradisce il vero e primo fine della normativa sui contratti, ovvero l'ultimazione dell'esecuzione ${ }^{56}$. Ma tant'è: gli ordinamenti democratici "consentono l'espressione di domande politiche di diversa natura, spesso confliggenti e, quindi, canonizzano interessi collettivi che le amministrazioni pubbliche devono curare, così aumentando la complessità degli apparati e dei processi di decisione ${ }^{\prime \prime 57}$.

Compreso quindi che semplificare fenomeni complessi non è facile né possibile ${ }^{58}$ - salvo abdicare al "momento amministrativo" (la c.d. Riforma Madia è andata per certi versi in questa direzione ${ }^{59}$ ) - ciò che rimane è rimuovere il "troppo", abbracciando una normativa minimale. Soluzione, questa, che aveva un nome (gold plating), ma che non è stata pienamente soddisfatta dal legislatore del 2016, il quale, come rilevato in dottrina, aveva effettivamente "complicato in misura significativa il Codice rispetto alla direttiva 2014/24 (con una scelta del tutto diversa da quella di molti altri paesi europei) $)^{160}$

Lo scoppio della crisi pandemica da COVID-19, da questo punto di vista, ha rappresentato una occasione. La Commissione europea, agli albori della

n. ${ }^{\circ} 1-2,2013$, pp. 15 e ss., e MARIO LiberTINI, Diritto della concorrenza dell'Unione europea, Milano: Giuffrè, 2014, pp. 2 e ss.

56 Stefano Vinti, "L'eterogenesi dei fini tradisce lo scopo ultimo e l'oggetto stesso della disciplina sui contratti pubblici", in federalismi.it, n. ${ }^{\circ} 12,2020$, pp. 252 e ss., e FABIO CinTIOLI, "Separare gli appalti dall'anticorruzione e disboscare le norme che bloccano le opere", in Il dubbio, 16 aprile 2020.

57 SABINO CASSESE, "Che cosa resta dell'amministrazione pubblica", in Rivista trimestrale di diritto pubblico, Fasc. 1, 2019, p. 3. Analoghe considerazioni sono svolte dall'A. nell'opera "Verso un nuovo diritto amministrativo?", in Giornale di. diritto amministrativo, n. ${ }^{\circ} 2,2016$, p. 12 e ss.

Va in questo senso Massimo OCCHIEnA, "Il coraggio di semplificare", in Diritto dell'economia., n. ${ }^{\circ} 102,2020$, pp. 1 e ss., il quale, in sostanziale continuità con quanto altrove affermato (Id., "Gli appalti pubblici e la semplificazione impossibile", in Dir. econ., vol. 26, n. ${ }^{\circ} 81,2013$, pp. 521 e ss.), sostiene che "non è possibile semplificare il settore de quo con interventi diretti a compendiare tutti i diversi interessi ed esigenze in gioco (concezione contabilistica, anticorruzione, concorrenza, tutela del lavoro, tutela dell'ambiente, ecc.), perché in questo modo giocoforza occorre prevedere disposizioni che tentino di coordinare elementi ispirati a fini talvolta persino contrastanti, generando così un eccesso di procedimentalizzazione e formalizzazione a detrimento della speditezza e dell'efficienza".

59 Cfr. MARCO BombardeLLI, "Il silenzio assenso tra amministrazioni e il rischio di eccesso di velocità nelle accelerazioni procedimentali", in Urbanistica e appalti., n. ${ }^{\circ}$ 7, 2016, pp. 758 e ss.

60 Marco Dugato, "Terremoto, ricostruzione e regole degli appalti", in Munus, n. ${ }^{\circ} 3$ 2017, p. 491. 
crisi, ha suggerito (ricordato?) agli Stati membri che "[i]l quadro europeo in materia di appalti pubblici offre agli acquirenti pubblici tutta la flessibilità necessaria per acquistare il più rapidamente possibile beni e servizi [e, qui si aggiunge, lavori] direttamente collegati alla crisi della COVID-19"61. Lo Stato italiano, da parte sua, seppur con un intervento a valenza interinale (sino al 31 dicembre 2021), ha poi scelto di tornare indietro, cioè di fare ritorno alla dir. 2014/24/Ue: il maggiore ricorso alla procedura negoziata senza previa pubblicazione del bando e, ciò che qui è più rilevante, il rilancio infrastrutturale passano infatti attraverso la "deroga ad ogni disposizione di legge", fatti salvi solo alcuni principi recati dal d.lgs. n. 50/2016 (artt. 30, 34 e 42), la disciplina in materia di subappalto, la normativa penale, il c.d. codice antimafia, nonché (e per l'appunto) "i vincoli inderogabili derivanti dall'appartenenza all'Unione europea, ivi inclusi quelli derivanti dalle direttive 2014/24/UE e 2014/25/UE" (cfr. art. 2, comma 4, d.1. 16 luglio 2020, n. 76, convertito con modificazioni dalla 1. 11 settembre 2020, n. 120). E, si badi, la deroga non vale solo per l'uso delle procedure negoziate per fronteggiare l'urgenza, ma anche e soprattutto per una consistente porzione del comparto infrastrutturale: i) "settori dell'edilizia scolastica, universitaria, sanitaria, giudiziaria e penitenziaria"; ii) "infrastrutture per attività di ricerca scientifica"; iii) "sicurezza pubblica, dei trasporti e delle infrastrutture stradali, ferroviarie, portuali, aeroportuali, lacuali e idriche, ivi compresi gli interventi inseriti nei contratti di programma ANAS-Mit 2016-2020 e RFI-Mit 2017-2021 e relativi aggiornamenti"; iv) "per gli interventi funzionali alla realizzazione del Piano nazionale integrato per l'energia e il clima (PNIEC)"; $v$ ) "interventi per la messa a norma o in sicurezza degli edifici pubblici destinati ad attività istituzionali, al fine [...] di recuperare e valorizzare il patrimonio esistente".

La semplificazione operata dal d.1. n. 76/2020 è stata certo ben maggiore

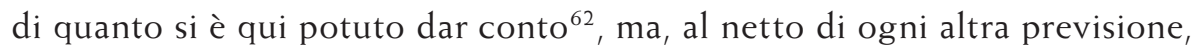
la deroga omnibus lì recata conferma il dato sopra accennato: semplificare le dinamiche contrattuali significa (o, almeno questo è quanto insegna l'esperienza più recente) conformare la disciplina al divieto di gold plating, accettando quindi le complicazioni recate dalle dir. 2014/24/Ue e 2014/25/Ue, senza prevederne di ulteriori ${ }^{63}$.

Ulteriori, piuttosto, devono essere le politiche di semplificazione rispetto a ciò che la normativa europea non disciplina. Un terreno elettivo è quello

61 Commissione Europea, Comunicazione della Commissione. Orientamenti della Commissione europea sull'utilizzo del quadro in materia di appalti pubblici nella situazione di emergenza connessa alla crisi della Covid-19, 2020/C 108 I/01.

62 Sul punto, cfr. Giuseppe Tropea e Annalaura Giannelli, "L'emergenza pandemica e i contratti pubblici: una 'semplificazione' in chiaroscuro, tra misure temporanee e prospettive di riforma strutturale", in Munus, Fasc.2, 2020, pp. 277 e ss.

63 Così anche MASSIMO OCCHIENA, "Il coraggio di semplificare". 
della responsabilità amministrativa, la quale, benché delineata alla stregua di "un punto di equilibrio tale da rendere, per dipendenti ed amministratori pubblici, la prospettiva della responsabilità ragione di stimolo, e non di disincentivo" ${ }^{164}$, è tradizionalmente percepita dall'agente pubblico come deterrente ${ }^{65}$ rispetto all'assunzione di scelte coraggiose (secondo la prospettiva del rischio) o, meglio a dirsi, strategiche ${ }^{66}$ (secondo la prospettiva che valorizza la matrice negoziale dell'operazione economica ${ }^{67}$, per quanto "collocata in un ambiente pubblicistico"68). Bene ha fatto quindi il legislatore italiano a limitare, pur temporaneamente (di nuovo, sino 31 dicembre 2021), la responsabilità amministrativa all'ipotesi del solo dolo, con esclusione, quindi, della colpa grave. Il che servirà sicuramente a "disinnescare i meccanismi cd. di burocrazia difensiva" 69 ed eventualmente a ripristinare la fiducia nell'uso del dialogo competitivo, ovvero di quella procedura che ad oggi non ha neppure autonomo risalto nelle tabelle sinottiche figurate dalle Relazioni annuali ANAC - e non a torto, considerando che nel 2019, l'Autorità affermava che "dal 2008 ad oggi sono state bandite 54 procedure di dialogo competitivo"70 - ma che invece sarebbe utilissima, specialmente nell'approntamento delle grandi infrastrutture pubbliche ${ }^{71}$.

\section{POTENZIALITÀ E CRITICITÀ NELLA GESTIONE DELLE RISORSE PER IL RILANCIO DELLE INFRASTRUTTURE}

Oltre alle potenzialità offerte dalla semplificazione, il rilancio infrastrutturale del Paese, come anticipato, deve essere sostenuto anche da un altro fattore: quello della destinazione delle risorse pubbliche.

64 C. cost., 20 novembre 1998, n. 371.

65 Come osservato in dottrina, non vi è affatto corrispondenza fra il "rischio reale" e quello "percepito" dall'agente pubblico: se il primo "assume una entità minima", il secondo invece "assume una consistenza molto elevata", GABRIELE BOTTINO, Riscbio e responsabilità amministrativa, Napoli: Editoriale Scientifica, 2017, p. 448.

66 Cfr. MASsimo RICCHI, "Negoauction, discrezionalità, dialogo competitivo e il nuovo promotore", in Le gare pubblicbe: il futuro di un modello, a cura di Gian Domenico Comporti, Napoli: Editoriale Scientifica, 2011, pp. 145 e ss.

67 Su cui si v. ENRICO GAbrielli, "L'operazione economica nella teoria del contratto", in Rivista Trimestrale di diritto processuale civ., n. ${ }^{\circ} 66$ (1), 2009, pp. 95 e ss.

68 Alberto Massera, Lo Stato che contratta e che si accorda. Vicende della negoziazione con le pp. AA., tra concorrenza per il mercato e collaborazione con il potere, Pisa: Edizioni Plus, 2011, p. 195.

69 Aldo SANDULLI, "Il codice dei contratti pubblici tra resistenze nazionali e spinte europee", in Munus, n. 3, 2019, p. 971.

70 AnAC, Relazione annuale 2018, Roma, Camera dei Deputati, 6 giugno 2019, 165.

71 Alberto Massera, Lo Stato che contratta e che si accorda, p. 278. 
Al fine di contrastare la grave crisi socio-economica a seguito della pandemia da COVID-19, l'Unione europea, su pressione di molti Stati membri tra cui l'Italia, ha deciso di promuovere un processo di ingenti investimenti per il rilancio economico dell'eurozona attraverso il c.d. Next Generation EU.

Come è noto, il Next Generation EU è un nuovo strumento temporaneo dell'Unione europea che, grazie alla raccolta di fondi sul mercato dei capitali, intende contribuire a riparare i danni economici e sociali causati dalla pandemia da COVID-19, attraverso l'erogazione di 750 miliardi di euro (390 di contributi a fondo perduto e 360 di prestiti) a favore dei vari Paesi europei. Il suo nucleo principale è rappresentato dal Dispositivo per la ripresa e la resilienza (RFF), volto a creare un'Europa più verde, digitale, resiliente e adeguata alle sfide presenti e future. La parte rimanente dei contributi verrà utilizzata su altri ambiti, tra cui React Eu, sviluppo rurale, Just transition fund. Data la gravissima situazione epidemica della primavera del 2020, con tutte le conseguenze in termini di decrescita dell'economia nazionale e del prodotto interno lordo, l'Italia sarà il maggiore beneficiario delle sovvenzioni grazie alla destinazione di circa 210 miliardi di euro, ripartiti in 82 miliardi di sovvenzioni e 128 miliardi di prestiti.

Ora, il Next Generation EU stimola riflessioni in relazione al grado di avanzamento nel processo di integrazione europea, sotto diversi profili: l'eventuale costruzione di un'autonomia fiscale unitaria $l_{i}$ 'evoluzione della politica delle istituzioni dell'eurozona, più "aperte" a svolgere un ruolo attivo nella ripresa economica, e dunque una funzione di rilievo quale soggetto "interventore" nell'economia, anche attraverso un ruolo più pervasivo degli organi politici dell'Unione (ad es. Commissione e Consiglio); la previsione di nuove strutture-ed eventualmente nuove procedure-a livello europeo che possano rafforzare i centri decisionali già esistenti, migliorandone il coordinamento con gli apparati nazionali.

In breve, il Next Generation EU pare rappresentare un punto di svolta nelle politiche dell'Unione, tradizionalmente "frugali" ed improntate al pareggio di bilancio nonché a stringenti limiti di spesa, preludendo ad una trasformazione dei modi di intervento europeo sugli equilibri di finanza pubblica. Questo programma favorevole alla ripresa e alla resilienza dovrebbe dunque costituire una occasione storica per agevolare un riassetto strutturale e funzionale dell'Unione Europea soprattutto attraverso un forte potenziamento delle opere e delle infrastrutture strategiche dell'euro-zona.

Tuttavia, per raggiungere questi obiettivi è necessario un'attivazione da parte di tutti i Paesi che aspirano ad ottenere i finanziamenti europei, chiamati a redigere un proprio piano di rilancio, con cui individuare in modo puntuale gli investimenti da promuovere, la loro fattibilità e le relative misure di monitoraggio, alla cui definizione è subordinato il rilascio delle sovvenzioni da parte dell'Unione europea. 
Nel periodo in cui si sta scrivendo (febbraio 2021), 1'Italia sta redigendo il Piano nazionale di Ripresa e Resilienza (PNRR), da presentare alla Commissione entro l'aprile del 2021, con l'obiettivo di indicare una serie di dati: le scelte compiute per l'individuazione degli impegni di spesa, da comunicare alle istituzioni europee inderogabilmente entro il 2023; l'effettivo impiego delle risorse; la previsione di eventuali meccanismi di controllo della messa in opera delle misure.

Grazie alla destinazione dei circa 210 miliardi di euro, il nostro Paese si troverà dunque a breve nella insolita situazione di avere risorse da spendere, diversamente da quanto accaduto negli ultimi decenni, durante i quali scarseggiava la liquidità necessaria per promuovere gli investimenti infrastrutturali e per garantire un'accurata manutenzione delle opere esistenti.

È dunque fondamentale che l'Italia definisca un efficace PNRR, descrivendo precisamente gli obiettivi ed i progetti con cui assicurare il rilancio infrastrutturale e le opere da realizzare sul territorio. Il punto focale della questione diviene, dunque, quello di indicare come saranno gestite le risorse, il che impone a sua volta di riflettere sul possibile nuovo ruolo dei poteri pubblici e sulle modalità di intervento nell'economia italiana.

In particolare, occorre considerare se lo Stato intenderà procedere direttamente alla realizzazione dei progetti infrastrutturali - in modo analogo a quanto accaduto tra gli anni Cinquanta e Sessanta del XX (infra par. 5) - o se, al contrario, si limiterà ad un ruolo di promotore e coordinatore degli investimenti, la cui attuazione verrà comunque demandata a soggetti esterni alla macchina amministrativa, dotati di conoscenze tecniche di cui essa non sempre dispone ${ }^{72}$.

A seconda della scelta che sarà compiuta, è evidente, muterà il rapporto tra le istituzioni e gli operatori privati i cioè, a breve lo Stato dovrà scegliere se tornare ad essere "imprenditore" come nella metà del secolo passato, ovvero ribadire la sua natura di "regolatore", delegando la materiale realizzazione delle infrastrutture agli operatori economici, limitandosi a selezionarli in ossequio ai principi di concorrenza, pubblicità, trasparenza, parità di trattamento e non discriminazione, in modo analogo a quanto già fatto dalla seconda metà degli anni Novanta sino ad oggi (per maggiori approfondimenti infra, par. 4-5) ${ }^{73}$.

Qualunque sarà la strada intrapresa, la redazione del PNRR, l'attuazione dei suoi obiettivi e la gestione delle risorse del Next generation EU da destinare all'ammodernamento del Paese si presentano come una sfida molto complessa per il governo.

72 Già la crisi economica del 2008 aveva condotto gli studiosi ad interrogarsi su questi temi. Si v., ad esempio, ANDREA PISANESCHI, Dallo Stato imprenditore allo Stato regolatore e ritorno?, Torino: Giappichelli, 2009.

73 Sulla contrapposizione tra Stato imprenditore e Stato regolatore, per tutti, AA. VV., La nuova costituzione economica, a cura di Sabino Cassese, Roma-Bari: Laterza, 2021. 
Si rilevano, infatti, alcune criticità che potrebbero rallentare il percorso di rilancio infrastrutturale, sintetizzabili in tre specifici fattori.

In primo luogo, sono prevedibili forti resistenze burocratiche se solo si pensa al tema della transizione digitale, la cui promozione si scontra con una cultura amministrativa (spesso) deficitaria, specialmente ricordando che i dipendenti degli uffici pubblici di frequente non vantano specifiche competenze in merito ${ }^{74}$. Questa situazione è ben visibile, ad esempio, con riferimento alle infrastrutture fisiche, considerando che l'amministrazione italiana sconta lo smantellamento dei corpi tecnici e l'inadeguatezza delle strutture organizzative, spesso prive delle necessarie competenze tecniche e da molto tempo disattente a percorsi "virtuosi" di reclutamento e crescita professionale, che ne comportano la scarsa capacità decisionale (sul punto v. anche infra par. 5).

In secondo luogo, emergono le probabili criticità derivanti dall'attuazione dei processi partecipativi della popolazione interessata alla progettazione di infrastrutture ${ }^{75}$. Il riferimento è, in particolare, alla capacità della popolazione di partecipare a queste decisioni, ma anche alla competenza dei responsabili e delle amministrazioni; alla lentezza attuativa del dibattito pubblico, e dunque a quelle forme di partecipazione affermatesi negli ultimi vent'anni, che hanno di frequente comportato rallentamenti nell'attuazione della decisione politica piuttosto che contribuire in modo efficace alla sua definizione ${ }^{76}$.

74 Gianluca SGUeO, "L'amministrazione digitale", in Giornale di dritto amministrativo, n. ${ }^{\circ}$ 1, 2016, pp. 114 e ss.; BRUNO CAROTTI, "L'amministrazione digitale: le sfide culturali e politiche del nuovo Codice", in Giornale di. diritto amministrativo, n. ${ }^{\circ} 1,2017$, pp. 7 e ss.; Piergiuseppe Otranto, "Decisione amministrativa e digitalizzazione della p.a.", in federalismi.it, n. ${ }^{\circ} 2,2018$.

75 Gregorio ARENA, "Introduzione all'amministrazione condivisa", in Studi parl. pol. cost., n. ${ }^{\circ} 3-4,1997$, pp. 29 e ss.; UMBERTO ALLEGRETTI, "La democrazia partecipativa in Italia e in Europa", in Riv. AIC, n. ${ }^{\circ} 1,2011$; UMBERTO AlLEGRETTI, "La democrazia partecipativa nella 'società liquida' e nella crisi della democrazia", in Partecipazione e comunicazione nelle nuove forme del piano urbanistico, a cura di Giancarlo Deplano, Monfalcone: Edicom Edizioni, 2009, pp. 17 e ss.; AA. VV., Per governare insieme: il federalismo come metodo. Verso nuove forme della democrazia, a cura di Gregorio Arena e Fulvio Cortese, Padova: Cedam, 2011; Luigi BobBio, "Dilemmi della democrazia partecipativa", in Dem. dir., n. ${ }^{\circ} 4,2006$, pp. 11 e ss.

76 Sul dibattito pubblico ed i suoi limiti attuativi, Umberto AlLEgretTi, "Senato delle regioni, organizzazione della giustizia dibattito pubblico sui grandi interventi infrastrutturali", in Quad. Riv. AIC, n. ${ }^{\circ} 2,2013$, p. 3 ss.; VALERIA DE SANTIS, "La nuova legge della regione Toscana in materia di dibattito pubblico regionale e promozione della partecipazione", in Osservatorio costituzionale, Fasc, 0, 2013. ANDREA Averard, "La decisione amministrativa tra dissenso e partecipazione", in Munus, n. ${ }^{\circ} 1,2018$, pp. 129 e ss.; ANDREA AVERARDI, "L'incerto ingresso del dibattito pubblico in Italia", in Giorn. dir. amm., n. ${ }^{\circ} 4,2016$, pp. 505 ss.; AlESSANDRO DI MARTINO, "Il dibattito pubblico per la realizzazione di grandi infrastrutture: quale ruolo per la partecipazione democratica?", 
In terzo luogo, non vanno dimenticate le difficoltà emergenti a livello di compatibilità e sostenibilità ambientale. Esse riguardano l'individuazione delle infrastrutture da ammodernare attraverso l'analisi del polinomio "asset-rischio-governance" e coinvolgono il problema della localizzazione delle nuove opere ${ }^{77}$, tema che va chiaramente coordinato con gli strumenti di analisi della pianificazione urbanistica e territoriale $\mathrm{e}^{78}$.

Tanto considerato, sembrano evidenti i problemi che accompagneranno la gestione delle risorse europee da parte dello Stato. È dunque bene capire se questo sarà in grado di gestire in via autonoma l'intero processo o se, al contrario, sia opportuno che continui a disporre di un sostegno esterno, cioè da parte del privato, nella realizzazione del processo di rilancio infrastrutturale. Il riferimento è al possibile ricorso agli strumenti di partenariato pubblico-privato (PPP), già utilizzati dai primi anni Duemila. Certo,

in Nuove aut., n. ${ }^{\circ} 3,2017$, pp. 531 e ss.; VANESSA MANZETTI, "Il 'dibattito pubblico' nel nuovo codice dei contratti", in federalismi.it, n. ${ }^{\circ}$ 5, 2018; Giovanna Mastrodonato, "Dibattito pubblico e partecipazione: le decisioni amministrative condivise nel Codice dei contratti pubblici", in Munus, n. ${ }^{\circ}$ 1, 2018, pp. 145 e ss.; Viviana MOLASCHI, "La democratizzazione delle decisioni 'science and technology based'. Riflessioni sul dibattito pubblico", in Nuove aut., Fasc. 3, 2017, pp. 463 e ss.; ANNA PIROzZOLI, "L'istituto del 'dibattito pubblico' sulle grandi opere", in Rass. parl., vol. 60, n. ${ }^{\circ} 2,2018$, pp. 455 e ss.; STEFANO ZUNARELLI, "Un nuovo strumento di prevenzione dei conflitti nella realizzazione delle grandi opere portuali: il dibattito pubblico", in Dir. mar., Fasc. 3, 2018, pp. 625 e ss.; IACOPO LuCA DianA, "La recente disciplina in tema di 'dibattito pubblico' sull'aggiudicazione di appalti e concessioni pubbliche", in Riv. giur. sarda, n. ${ }^{\circ} 3$, pt. II, 2016, pp. 111 e ss.

77 GiUSEPPE PIPERATA, "Infrastrutture e crescita economica: alla ricerca di nuovi modelli", pp. III e ss.; RUGGIERO DiPACE, "La logica della prevenzione nella disciplina della programmazione e progettazione di lavori pubblici", in Riv. giur. edil., n. ${ }^{\circ} 6,2018$, pp. 445 e ss.; SANDRO AmOrosino, "Regolazione e programmazione delle infrastrutture", in Urb. app., Fasc. 1, 2019, pp. 46 e ss.; SUSANnA SCREPANTI, "I tempi delle opere pubbliche e la relatività del tempo nel diritto amministrativo", in Riv. trim. dir. pubbl., vol. 69, n. ${ }^{\circ}$ 4, 2019, pp. 1189 e ss.; AA. VV., "Pianificazione, programmazione, localizzazione e progettazione, servizi e concorsi di progettazione", in Trattato sui contratti pubblici, a cura di Maria Alessandra Sandulli e Rosanna de Nictolis, tomo IV, Milano: Giuffrè, 2019; FRANCO PELLIZZER, "La programmazione e la progettazione", in Diritto dei contratti pubblici, a cura di Franco Mastragostino, Torino: Giappichelli, 2019, pp. 239 e ss.; Girolamo Sciullo, "Concertazione istituzionale e localizzazione delle grandi opere", in Giorn. dir. amm., vol. 10, 2004, pp. 1088 e ss.; ROBERTO GALLIA, "La spesa per infrastrutture degli Enti locali: l'efficacia della programmazione condizionata dalle semplificazioni procedimentali", in Riv. giur. mezz., Fasc. 2, 2019, pp. 379 e ss.; Stefano Civitarese MatteucCI, "Semplificazione ed autocoordinamento nei procedimenti di localizzazione di opere statali", in le Regioni, vol. 1, 1995, pp. 99 e ss.

78 Sandro AmOrosino, "Sviluppo economico e governo del territorio", in Rivista giuridica edilizia, n. ${ }^{\circ}$ 5, 2015, pp. 187 e ss.; ROSANNA DE NiCTOLIS, "La funzione di pianificazione urbanistica e la localizzazione e realizzazione di opere pubbliche", in Cons. St., vol. 55, Fasc. 11, 2004, pp. 2263 e ss. 
la partnership ha spesso dovuto scontare importanti criticità organizzative (infra par. 4); nondimeno, anche in ragione delle competenze che potrebbe apportare, un rinnovato ruolo dell'operatore economico privato a cui affidare la progettazione, costruzione e manutenzione delle opere, con l'assunzione dei relativi rischi di gestione, potrebbe non rappresentare una scelta a priori errata, purché vengano posti i dovuti correttivi per impedire di ricadere negli errori del passato.

\section{IL PROBLEMATICO RICORSO AI PPP NELLA REALIZZAZIONE E GESTIONE DELLE INFRASTRUTTURE}

I PPP costituiscono un complesso fenomeno di natura socio-politica, giuridica ed economica ${ }^{79}$, a cui afferiscono vari istituti (concessioni, società partecipate, etc.), regolati da fonti sovranazionali e nazionali, che trovano la propria peculiarità nella collaborazione tra le amministrazioni e i privati, allo scopo di soddisfare un interesse della collettività tramite il ricorso alla formula contrattuale più adeguata ${ }^{80}$. I motivi del ricorso alla cooperazione sono sostanzialmente due: la perdurante carenza di risorse per la realizzazione di infrastrutture da parte delle amministrazioni e l'affidamento alle competenze tecniche del privato, che spesso dispone di conoscenze maggiori rispetto a quelle degli enti pubblici ${ }^{81}$.

In ambito europeo, l'Unione è intervenuta sul tema, in principio, con il Libro verde della Commissione europea del 30 aprile $2004^{[82]}$ ed il documento Eurostat dell'11 febbraio $2004^{[83]}$, poi con la direttiva con la comunicazione del 5 febbraio 2008 e, infine, con la direttiva 2014/23/Ue. In tutte queste circostanze, gli atti normativi europei non hanno individuato una nozione univoca di PPP, concetto rimasto sempre piuttosto vago, tanto è vero che è "a livello comunitario non esiste una normativa specifica sulla costituzione

79 Alfredo FiORITTO, "L'amministrazione negoziale: modelli di partenariato e problemi di applicazione", in Nuove forme e nuove discipline del partenariato pubblico privato, a cura di Alfredo Fioritto, Torino: Giappichelli, 2017, p. 55.

80 La letteratura sui PpP è estremamente vasta, per cui nel corso del lavoro saranno considerati i principali contributi sul tema. Per un recentissimo studio sui principali caratteri dell'istituto, SARA VAlaguzZa e Eduardo PARISI, "Ricerca sull'identità giuridica del partenariato pubblico-privato", in Munus, n. ${ }^{\circ} 1,2020$, pp. 1 e ss.

81 Mario Pilade Chiti, "Il partenariato pubblico privato e la nuova direttiva concessioni", in Finanza di progetto e partenariato pubblico privato, a cura di Gian Franco Cartei e Massimo Ricchi, Napoli: Editoriale scientifica, 2015, pp. 3 y ss.

82 Libro verde relativo ai partenariati pubblico-privati ed al diritto comunitario degli appalti pubblici e delle concessioni, punto 1.1.3.

83 Si fa riferimento al documento "Treatment of public-private parnerships". 
dei PPP" ${ }^{\prime 84}$ Tuttavia, la Commissione ha precisato i contenuti giuridici del concetto, individuandoli nel trasferimento del c.d. rischio operativo - di costruzione, di domanda e di disponibilità dell'opera - in capo al privato nella costruzione e gestione dell'opera stessa ${ }^{85}$.

Da quanto premesso, è evidente la matrice economica dei PPP $^{86}$, a motivazione dell'attenzione riservata al tema dalle istituzioni europee, interessate ad una disciplina in armonia con i principi di parità di trattamento, pubblicità, trasparenza e concorrenza, così da legittimare l'affidamento dell'attività a favore dell'operatore ${ }^{87}$. Infatti, l'affidamento in concessione di un'attività economica al privato - quale è la costruzione e gestione di un'opera e la resa del relativo servizio - impone il rispetto di rigorose regole concorrenziali per evitare discriminazioni nei confronti dei potenziali operatori che aspirano a contrattare con l'amministrazione.

Anche il legislatore italiano è intervenuto sulla materia, recependo la normativa europea, eppure adeguandovisi solo in parte. Infatti, gli artt. 180 ss., d.lgs. n. 50/2016, regolano il ricorso ai PPP, includendo però in tale elenco anche alcune tipologie contrattuali a carattere sociale (es. il baratto amministrativo, sussidiarietà orizzontale, etc.), obiettivamente eterogenei dalle concessioni di lavori e di servizi, non configurando un'attività economica. Pertanto, il concetto italiano di PPP è ben più ampio rispetto a quello europeo $^{88}$, che invece si esaurisce nelle concessioni di lavori e servizi ${ }^{89}$.

È però evidente che, soffermandosi in questa sede sulle vicende della crisi infrastrutturale, ben evidenziate dalla pandemia, sia opportuno rivolgere lo

Punto 2.1. della comunicazione.

Cfr. Aldo TRAVI, "Il partenariato pubblico-privato: i confini incerti di una categoria", in Negoziazioni pubbliche, a cura di Maurizio Cafagno, Alessandro Botto, Gianfrancesco Fidone e Gabriele Bottino, Milano: Giuffrè, 2013, pp. 10 e ss. Più di recente, i motivi citati del ricorso al PPP sono elencati anche da CLAUDio CONTESSA, "PPPC: modello generale, in Concessioni di lavori e servizi, partenariati precontenzioso e contenzioso", in Trattato sui contratti pubblici, V, diretto Maria Alessandra Sandulli e Rosanna de Nictolis, Milano: Giuffrè, 2019, pp. 208-209. Per la definizione dei rischi di costruzione, di disponibilità e di domanda, si vedano rispettivamente le lett. aaa), bbb) e ccc), dell'art. 3, comma 1, d.lgs. n. 50/2016.

86 Così anche MARCo DuGATO, "Il partenariato pubblico-privato: origine dell'istituto e sua evoluzione", in La collaborazione pubblico-privato e l'ordinamento amministrativo, a cura di Franco Mastragostino, Torino: Giappichelli, 2011, pp. 55 e ss.

87 Il riferimento è alla comunicazione interpretativa della Commissione sulle concessioni nel diritto comunitario del 29 aprile 2000. Circa i principi che regolano la concessione di attività economiche dal pubblico al privato, si veda il par. 3 della comunicazione.

SARA VAlaguzZa e EdUARDo PARISI, "Ricerca sull'identità giuridica del partenariato pubblico-privato"; GIUSEPPE PIPERATA, "Introduzione", in La collaborazione pubblico-privato el'ordinamento amministrativo, a cura di Franco Mastragostino, Torino: Giappichelli, 2011, p. 10.

89 Mario PILADE CHITI, "Il partenariato pubblico privato e la nuova direttiva concessioni", pp. 12-13. 
studio al rapporto partenariale nella sua accezione più ristretta di origine europea, per poi verificare se il privato possa rivelarsi ancora oggi utile nel programma di potenziamento delle opere pubbliche di carattere strategico o, al contrario, il suo ruolo sia divenuto superfluo stante le ingenti risorse europee destinate all'Italia dal c.d. Next Generation EU.

Il dubbio è ancor più legittimo se si pensa al fatto che il ricorso ai PPP negli ultimi anni è diminuito, soprattutto con riferimento all'area dell'eurozona $^{90}$, nonostante i dati siano in parte più incoraggianti per l'Italia. Il nostro paese ha infatti avviato un percorso di graduale ma crescente utilizzo dello strumento nel corso dell'ultimo quindicennio, durante il quale sono stati soprattutto i comuni ad avere fatto ricorso alla formula partenariale per opere di dimensioni contenute (edilizia residenziale e sociale, impianti sportivi) ${ }^{91}$.

Questo anche perché l'utilizzo dei PPP per le concessioni di lavori e servizi sconta per le opere di grandi dimensioni difficoltà di vario genere, che vanno dalla complessa gestione della procedura ad evidenza pubblica ai problemi legati alla costruzione del vincolo contrattuale.

Con riferimento alla gara per l'aggiudicazione, è necessario che le concertazioni con l'operatore economico siano svolte da funzionari e tecnici amministrativi dotati di una preparazione e di un "commitment pubblico" forti ${ }^{92}$, con elevate capacità manageriali, che nei fatti sono spesso mancate ${ }^{93}$. Inoltre, la complessità delle operazioni non ha nemmeno agevolato la celere conclusione dei procedimenti, causando invero importanti rallentamenti nella conclusione dei PPP, i cui tempi di aggiudicazione sono risultati spesso eccessivi ${ }^{94}$. Al prolungamento dei tempi conseguono rischi progettuali maggiori per il concessionario, che generalmente determinano una revisione al rialzo del compenso richiesto, e dunque maggiori oneri per la collettività, una minore convenienza del ricorso ai ppp ed un più elevato rischio di insuccesso delle operazioni ${ }^{95}$.

90 Rapporto Epec anno 2019, per la cui lettura ed un'analisi più approfondita dei dati si rinvia all'indirizzo https://www.eib.org/en/search.htm?q=market+update\&sortColu$\mathrm{mn}=$ relevant\&sortDir $=$ desc \& pageNumber $=0$ \& itemPerPage $=10 \&$ pageable $=$ true $\&$ lan guage $=\mathrm{EN} \&$ default Language $=\mathrm{EN} \&$ orContent $=$ true $\&$ orPageType $=$ true

91 Dipartimento per la programmazione economica, Partenariato pubblico privato e finanza di progetto, in www.programmazioneeconomica.gov.it

92 Corte conti, sez. riun. contr., Rapporto 2017 sul coordinamento della finanza pubblica, 282, in www.cortedeiconti.it

93 Corte conti europea, Partenariati pubblico-privato nell'UE: carenze diffuse e benefici limitati, relazione speciale n. 09/2018,60 ss.

94 Cfr. il rapporto del Dipe Partenariato pubblico e privato e finanza di progetto, par. 1.2.; Corte conti europea, Partenariati pubblico-privato nell'UE, 28-40.

95 Rapporto I comuni italiani e il Partenariato Pubblico Privato, a cura di Carla Giorgio, Giorgia Marinuzzi e Walter Tortorella, documento di analisi "Ufficio valutazione impatto" del 
Anche le difficoltà nella costruzione del vincolo contrattuale sono di grande rilievo, perché è in questo frangente che pubblico e privato stabiliscono le tariffe per la gestione del servizio e le modalità di fruizione dell'opera.

Un accordo tra le parti è efficace se soddisfa diversi aspetti, quali ad esempio la definizione di formule contrattuali standard e di linee guida per la redazione dei capitolati delle partnership, l'effettivo trasferimento del rischio operativo in capo al privato, la previsione un'adeguata fase di monitoraggio, cioè tutti fattori non sempre oggetto di adeguata considerazione, per cui le prestazioni dell'operatore sono spesso risultate poco soddisfacenti ${ }^{96}$. Del resto, l'importanza di una efficace stesura dei contratti si spiega anche alla luce di questioni eminentemente finanziarie, al fine di evitare che i PPP, inizialmente registrati come off-balance (e quindi non contabilizzati tra le spese dello Stato), vengano in seguito riclassificati come on-balance, con gravi conseguenze sul deficit pubblico ${ }^{97}$.

Considerata l'importanza della questione, il Ministero dell'economia e delle finanze ha prodotto delle linee guida - su cui è intervenuto anche un parere del Consiglio di $\operatorname{Stato}^{98}$ - per la redazione di contratti di PPP, soffermandosi sulle maggiori criticità: l'effettivo trasferimento del rischio operativo in capo all'operatore economico ${ }^{99}, l^{\prime}$ eventuale esclusione del contributo pubblico per la realizzazione delle c.d. opere fredde ${ }^{100}$, la revisione del piano economico finanziario (pef), la possibilità di apportare delle modifiche al contratto ${ }^{101}$.

L'intervento, mosso dalle migliori intenzioni, intende delineare degli indirizzi univoci per la regolazione standardizzata di rapporti contrattuali,

Senato della Repubblica, 2018, 30, individua, ad esempio, una media di 302 giorni per aggiudicare bandi relativi a finanze di progetto.

96 MARCO MACCHIA, "La regolamentazione degli affidamenti da parte dei concessionari", in Rivista italiana diritto pubblico comparato, n. ${ }^{\circ} 1,2018$, pp. 153 e ss.

97 La relazione annuale dell'Autorità nazionale anticorruzione (ANAC) del 2020 ha rimarcato l'importanza di una idonea stesura dei contratti di partenariato anche per ragioni strettamente finanziarie: Anac, Relazione annuale 2020, pp. 40-41 e 301-302.

98 Cons. St., parere 28 aprile 2020, n. 823.

99 Il trasferimento del rischio operativo in capo all'aggiudicatario è condizione necessaria perché l'operazione possa essere contabilizzata al di fuori dello stato patrimoniale delle amministrazioni, senza essere quantificata in termini di indebitamento. Cfr. GIAN FRANCO CARTEI, "Rischio operativo, equilibrio economico-finanziario e disciplina delle sopravvenienze", in Finanza di progetto e partenariato pubblico privato, a cura di Gian Franco Cartei e Massimo Ricchi. Napoli: Editoriale scientifica, 2015, p. 28.

100 Le opere fredde sono incapaci di generare reddito attraverso la fruizione da parte di terzi, per cui l'operatore economico ottiene la remunerazione direttamente dall'ente pubblico (ne sono un esempio gli ospedali, le scuole, le carceri).

101 Le linee guida redatte dal Ministero sono consultabili all'indirizzo https://www.rgs. mef.gov.it/VERSIONE-I/attivita_istituzionali/attivit_trasversali/ppp/contratti_standard_e_linee_guida/ 
così da definire un valido vincolo sinallagmatico, la cui efficacia è spesso minacciata sia da un automatico (e sproporzionato) sostegno pubblico agli investimenti del concessionario ${ }^{102}$ sia dalla revisione dei prezzi in suo favore

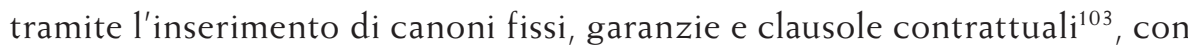
conseguente aumento dei costi per le amministrazioni concedenti. In breve, la definizione di clausole contrattuali certe è funzionale ad evitare che si verifichi l'ipotesi meno gradita: il mancato trasferimento di un effettivo rischio operativo in capo al privato a causa del sostegno offertogli dall'amministrazione, preoccupata dal fatto che le oscillazioni e le contrazioni del mercato rendano conveniente per l'operatore l'abbandono del servizio, con evidenti ricadute significative sulla collettività che ne stava beneficiando.

Tuttavia, ancora oggi, le previsioni delle linee guida scontano alcune difficoltà di attuazione, se non altro perché non sono una fonte normativa di carattere cogente, per cui permane un problema di soluzione delle criticità sin qui segnalate, che si riflette sulle modalità di ricorso ai PPP ${ }^{104}$.

Altro profilo di contraddizione nell'utilizzo della formula partenariale si riscontra poi nel fatto che il diritto nazionale, per fronteggiare l'emergenza Covid, abbia promosso diverse normative spesso in contrasto con le regole europee della concorrenza, facendo dunque dubitare della tenuta di un modello basato sul libero mercato ${ }^{105}$.

Per queste varie ragioni, è legittimo chiedersi se sia utile ricorrere ai PPP nel progetto di rilancio infrastrutturale o se, al contrario, questo strumento possa ritenersi oggi superato, considerando anche (e soprattutto) le risorse europee a disposizione del Paese. In altre parole, occorre valutare se lo Stato regolatore che ha avuto grande fortuna in Europa dagli anni Novanta in poi debba lasciare spazio al ritorno dello Stato imprenditore, la cui affermazione - quantomeno in Italia - si è avuta tra gli anni Trenta e Sessanta del XX secolo ${ }^{106}$.

102 Mariano Protto e LuCa Mazzeo, Project financing $e$ infrastrutture strategiche: aspetti di criticità dell'attuale sistema e loro (possibile) superamento alla luce della direttiva 2014/23/Ue, in: AA. VV., Finanza di progetto e partenariato pubblico privato, 2015, p. 147.

103 Rileva questi problemi Corte conti, sez. aut., 23 giugno 2017, n. 15.

104 Sulle difficoltà di attuazione della soft law, ANA LuCIA VALVO, "'Soft law' e (in)certezza del diritto con particolare riguardo all'ordinamento dell'Unione europea", in Riv. coop. giur. internaz., n. ${ }^{\circ} 64,2020$, pp. 22 e ss.

105 Così Giuliano Fonderico, "Finanziamenti a imprese e lavoratori autonomi", in Giornale di. diritto amministrativo, $\mathrm{n} .{ }^{\circ} 5,2020$, pp. 568 e ss.

106 Sulla contrapposizione tra Stato regolatore e Stato imprenditore, per tutti, AA. VV., La nuova Costituzione economica. 


\section{PER CONCLUDERE: UN RITORNO DELLO STATO IMPRENDITORE O UNA (RI) CONFERMA DELLO STATO REGOLATORE?}

Nel quadro sin qui delineato, tra crisi infrastrutturale, politiche di semplificazione e destinazione dei finanziamenti, rimane da considerare il rapporto che lo Stato potrà instaurare con il privato, non avendo bisogno della sua liquidità per attuare il piano di rilancio.

Cassa depositi e prestiti $(\mathrm{CdP})-$ l'istituzione finanziaria pubblica che funge da tesoriere dello Stato, controllata dal Ministero dell'economia e delle finanze - rivestirà con ogni probabilità una funzione primaria nella gestione delle risorse e nella programmazione degli interventi ${ }^{107}$. Del resto, la centralità dello Stato nella ricostruzione del tessuto economico-imprenditoriale non rappresenterebbe un fatto nuovo, perché già nel secondo Dopoguerra

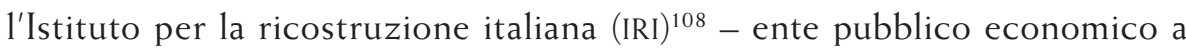
cui fu affidata la costruzione delle maggiori opere pubbliche sul territorio nazionale - svolse un'attività fondamentale per il potenziamento infrastrutturale e l'ammodernamento del Paese, risultando uno dei protagonisti del "miracolo economico italiano"109, secondo una logica di programmazione pubblica dell'economia110.

A ben vedere, però, $\mathrm{CdP}$ ha una natura di intermediario finanziario e non di decisore ed attuatore di politiche di sviluppo economico ed infrastrutturale, come era invece IRI (la cui attività è cessata nel 2002), per cui difficilmente

107 Per alcuni approfondimenti su CdP, MARCO MACCHIA, "La Cassa depositi e prestiti tra i diritti e le reti di nuova generazione", in Munus, n. ${ }^{\circ}$ 1, 2014, pp. 93 e ss.; ANDREA Averard, Potere pubblico e politiche industriali, Napoli: Jovene, 2018.

108 VinCENZO M. SBRESCIA, "Alberto Beneduce e le trasformazioni del modello di interventi pubblico in economia: la vicenda dello Stato imprenditore ed il ruolo dell'Iri a quindici anni dalla sua messa in liquidazione", in Riv. giur. mezz., v. 1, 2015, pp. 761 e ss.

109 Tuttavia, IRI creò anche numerosi problemi in quanto passò dall'essere un vanto del sistema italiano a divenire un peso insostenibile per le finanze pubbliche: l'ostacolo all'affermazione dell'impresa privata, il metodo anti-economico della programmazione, l'eccessivo assistenzialismo della gestione imprenditoriale, pronta a coprire ogni perdita finanziaria delle aziende pubbliche, la creazione di posti di lavoro strettamente legata ad interessi di partito e logiche clientelari, sono tutti elementi che hanno causato la crescita vertiginosa del debito pubblico, di cui ancora oggi stiamo pagando. Per una lettura, ANDREA PISANESCHI, Dallo Stato imprenditore allo Stato regolatore e ritorno?, pp. 22-24, 31,34-35, evidenzia i problemi dell'Iri sopra elencati, già avvertiti a fine anni Quaranta e primi anni Cinquanta, sintetizzabili nell'eccessiva disinvoltura della propria attività imprenditoriale, giunta sino alla costruzione di alberghi. Considerazioni sulle progressive inefficienze dell'IRI, VINCENZO M. SBRESCIA, "Alberto Beneduce e le trasformazioni del modello di interventi pubblico in economia: la vicenda dello Stato imprenditore ed il ruolo dell'Iri a quindici anni dalla sua messa in liquidazione", pp. 5-6.

110 Si badi, di programmazione, non di pianificazione, modello tipico dei Paesi socialisti. 
potrebbe gestire dal punto di vista operativo l'opera di ricostruzione ${ }^{111}$. La mancanza di un ente con quelle caratteristiche è di ostacolo alla riproposizione di un modello di Stato imprenditore affermatosi nel secolo scorso.

Il corollario di queste considerazioni è che il patrimonio conoscitivo del privato non sembra rinunciabile a priori.

Come già osservato, i partenariati non sono stati utilizzati solo per sopperire alla mancanza di risorse pubbliche, ma anche per fare fronte all'inadeguata preparazione delle amministrazioni, i cui corpi tecnici sono stati "smantellati", depotenziati e depauperati delle proprie funzioni decisorie nel corso dei decenni del secolo scorso ${ }^{112}$. Pertanto, la disponibilità in capo allo Stato di ingenti risorse non risolverebbe comunque l'endemico problema della debolezza della macchina amministrativa, le cui competenze tecniche sono state gradualmente dismesse - anche a livello centrale - e devono perciò ricercarsi all'esterno - e cioè nei privati - non potendosi ricostituire nel breve periodo.

Certo, sarebbe facile obiettare che lo strumento partenariale perderebbe egualmente di importanza poiché il processo di rilancio infrastrutturale potrebbe attuarsi attraverso i soli appalti di lavori. Nemmeno questa lettura, però, convince del tutto per due motivi: i privati sarebbero pur sempre presenti; se, come detto, l'amministrazione ha in larga parte perso le proprie competenze tecniche e non è più in grado di fare, si corre il forte rischio che non riesca neppure a definire unilateralmente strategie efficaci, delegare, controllare ed, eventualmente, correggere (salvo ricorso ad oggi molto incerto alle procedure di dialogo competitivo $)^{113}$.

Peraltro, è evidente che la risposta al problema della crisi infrastrutturale non potrà essere quella di una riproposizione nell'uso dei PPP come promossi negli ultimi quindi anni, considerando le diverse criticità sopra segnalate. Nondimeno, l'impressione è che l'apporto del privato specializzato non costituisca un fattore a cui si possa rinunciare in toto, soprattutto in ragione delle numerose opere che il governo sta programmando, e che difficilmente

111 Maria Teresa Salvemini, "La posizione di Cassa depositi e prestiti", in Rivista giuridica mezzogiorno, n. ${ }^{\circ} 1,2015$, pp. 681 e ss.

112 Guido Melis, "La fuga dall'amministrazione. Ascesa e declino dei tecnici nell'amministrazione dell'Italia unita", in Rivista trimestrale di diritto pubblico, n. ${ }^{\circ} 2,2013$, pp. 469 e ss.; Guido MELIS, "Culture dei tecnici e amministrazione nell'Italia di ieri e di oggi", in Rivista giuridica Mezzogiorno, n. ${ }^{\circ} 2,2019$, pp. 291 e ss.; SABINO CASSESE, "Che cosa resta dell'amministrazione pubblica?"; LUIGI FIORENTINO, "I corpi tecnici delle amministrazioni: problemi attuali", in Rivista trimestrale diritto pubblico, n. ${ }^{\circ} 2,2013$, pp. 479 e ss

113 È questo il senso delle riflessioni di LORENZO SALTARI, "Che resta delle strutture tecniche nell'amministrazione pubblica italiana?", in Rivista trimestrale di diritto pubblico., n. ${ }^{\circ} 1$, 2019, pp. 249 e ss. Giunge alle stesse conclusioni ALDO SANDULLI nella citata Intervista su La Repubblica di V. Conte, 12 febbraio 2021. 
verrebbero realizzate integralmente in autonomia dallo Stato, per ragioni sia di tempo sia di competenze tecniche.

Se, dunque, non pare saggio derubricare i PPP come strumento superato per il solo fatto che il Paese disporrà delle risorse europee, si ritiene che la sfida del rilancio consisterà nel non ripetere gli errori del passato, causati sia dalle nefaste conseguenze dell'eccessivo interventismo dello Stato nella programmazione economica sia dai limiti nel ricorso alle formule partenariali. L'obiettivo deve dunque essere quello di far convivere nuovi e vecchi strumenti: dirigismo, programmazione, tutela della concorrenza, controlli e standardizzazione dei rapporti contrattuali.

Considerata la questione sotto queste prospettive, sembra che lo Stato regolatore, pur rivedibile nel suo funzionamento, non debba lasciare spazio ad un ritorno dello Stato imprenditore, così come conosciuto in passato, perché la replica di quell'esperienza condurrebbe con sé il rischio - non sostenibile - di una ulteriore crescita di deficit pubblico.

L'unica cosa di cui il Paese non ha bisogno.

\section{BIBLIOGRAFIA}

AA. VV. "Pianificazione, programmazione, localizzazione e progettazione, servizi e concorsi di progettazione". In Trattato sui contratti pubblici, a cura di Maria Alessandra Sandulli e Rosanna de Nictolis, tomo IV. Milano: Giuffrè, 2019.

AA. VV. La nuova costituzione economica, a cura di Sabino Cassese. Roma-Bari: Laterza, 2021.

AA. VV. Per governare insieme: il federalismo come metodo. Verso nuove forme della democrazia, a cura di Gregorio Arena e Fulvio Cortese. Padova: Cedam, 2011.

Agenzia Per la Coesione Territoriale. Rapporto sui tempi di attuazione delle opere pubbliche, Roma, 2018.

Allegretti, Umberto. "La democrazia partecipativa in Italia e in Europa". Riv. AIC, n. ${ }^{\circ} 1,2011$.

AlLEGRETTI, UMBERTO. "La democrazia partecipativa nella 'società liquida' e nella crisi della democrazia". In Partecipazione e comunicazione nelle nuove forme del piano urbanistico, a cura di Giancarlo Deplano. Monfalcone: Edicom Edizioni, 2009.

Allegretti, Umberto. "Senato delle regioni, organizzazione della giustizia dibattito pubblico sui grandi interventi infrastrutturali". Quad. Riv. AIC, n. ${ }^{\circ} 2,2013$. 
Amorosino, SANDRO. "Regolazione e programmazione delle infrastrutture". Urb. app., Fasc. 1, 2019.

Amorosino, Sandro. "Sviluppo economico e governo del territorio". Rivista giuridica edilizia, $\mathrm{n}^{\circ} .5,2015$.

AnCe-Direzione Affari Economici e Centro Studi. Osservatorio congiunturale sull'industria delle costruzioni, Roma, 2020.

Arena, Gregorio. "Introduzione all'amministrazione condivisa". Studi parl. pol. cost., n. ${ }^{\circ} 3-4,1997$.

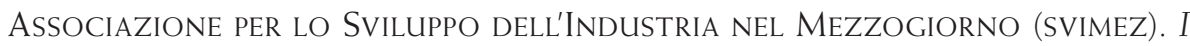
divari infrastrutturali e la spesa per opere pubbliche: la mancata attuazione della perequazione infrastrutturale, 2019.

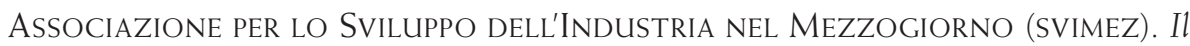
Mezzogiorno nella storia economica d'Italia. Una questione aperta, Roma, 2017.

AVERARDi, ANDREA. "L'incerto ingresso del dibattito pubblico in Italia". Giornale di diritto amministrativo, $\mathrm{n}^{\circ}$ 4, 2016.

AVERARDi, ANDREA. "La decisione amministrativa tra dissenso e partecipazione". Munus, n. ${ }^{\circ} 1,2018$.

Averardi, Andrea. Potere pubblico e politiche industriali. Napoli: Jovene, 2018.

Bobbio, Luigi. "Dilemmi della democrazia partecipativa". Dem. dir., n. o 4, 2006.

BOMBARDELLI, MARCO. "Il silenzio assenso tra amministrazioni e il rischio di eccesso di velocità nelle accelerazioni procedimentali". Urbanistica e appalti, n. ${ }^{\circ}$ 7, 2016.

BOneTTI, TOMmaso. "Il trasporto pubblico locale nel prisma della mobilità sostenibile". Diritto. amministrativo, v. 3, 2020.

Boscolo, Emanuele. "Consultazioni preliminari di mercato e possibili limitazioni alla concorrenza". Giur. it., n. ${ }^{\circ}$ 1, 2020.

BOtTINO, GABRIELE. Rischio e responsabilità amministrativa. Napoli: Editoriale Scientifica, 2017.

Busetti, Fabio, Cristina Giorgiantonio, Giorgio Ivaldi, Sauro Mocetti, Alessandro Notarpietro e Pietro Tommasino. "Capitale e investimenti pubblici in Italia: effetti macroeconomici, misurazione e debolezze regolamentari". Banca d'Italia-Eurosistema. Questioni di Economia e Finanza (Occasional Papers), n. ${ }^{\circ}$ 520, ottobre 2019. 
CAIA, GiUSEPPE. "Criterio di economicità e servizi pubblici locali nella prospettiva della XVIII legislatura repubblicana". In Diritto amministrativo e società civile, t. I, Studi introduttivi. Bologna: Bononia University Press, 2018.

CaiA, GiUseppe. "Il trasporto pubblico locale come paradigma del servizio pubblico (disciplina attuale ed esigenze di riordino)". Rivista AIC, n. ${ }^{\circ}$ 3, 2018.

Cammelli, Marco. "Istituzioni pubbliche e sviluppo economico locale". Ist. fed. n. ${ }^{\circ} 2,2009$.

CarotTI, BRUnO. "Lamministrazione digitale: le sfide culturali e politiche del nuovo Codice". Giornale di. diritto amministrativo, n. ${ }^{\circ}$ 1, 2017.

CARTEI, Gian Franco. "Rischio operativo, equilibrio economico-finanziario e disciplina delle sopravvenienze". In Finanza di progetto e partenariato pubblico privato, a cura di Gian Franco Cartei e Massimo Ricchi. Napoli: Editoriale scientifica, 2015

CASSESE, Sabino. "Che cosa resta dell'amministrazione pubblica?". Rivista trimestrale di diritto pubblico, Fasc. 1, 2019.

CASsese, Sabino. "Dallo sviluppo alla coesione. Storia e disciplina vigente dell'intervento pubblico per le aree insufficientemente sviluppate". Rivista Trimestrale di diritto pubblico, n. ${ }^{\circ} 2,2018$.

Cassese, Sabino. "Verso un nuovo diritto amministrativo?". Giornale di diritto amministrativo, n. ${ }^{\circ} 2,2016$.

Cassese, Sabino. Il diritto amministrativo: storia e prospettive. Milano: Giuffrè, 2010.

Chiti, Mario Pilade. "Il partenariato pubblico privato e la nuova direttiva concessioni". In Finanza di progetto e partenariato pubblico privato, a cura di Gian Franco Cartei e Massimo Ricchi. Napoli: Editoriale scientifica, 2015.

Chiti, Mario PILAde. "Principi". In Trattato sui contratti pubblici, t. I, diretto Maria Alessandra Sandulli e Rosanna de Nictolis. Milano: Giuffrè, 2019.

Cintioli, Fabio. "Separare gli appalti dall'anticorruzione e disboscare le norme che bloccano le opere", Il dubbio, 16 aprile 2020.

Civitarese Matteucci, Stefano. "Semplificazione ed autocoordinamento nei procedimenti di localizzazione di opere statali". le Regioni, vol. 1, 1995.

COMPORTI, GiAn DOMENICO. "La flessibilità nelle negoziazioni pubbliche: questa sconosciuta". Dir. soc., n. ${ }^{\circ}$ 2, 2017. 
CONTESSA, ClaUdio. PPPC: modello generale, in Concessioni di lavori e servizi, partenariati precontenzioso e contenzioso, t. V, in Trattato sui contratti pubblici, diretto da Maria Alessandra Sandulli e Rosanna de Nictolis. Milano, Giuffrè, 2019.

CROSETti, AlESSANDRO. "Dalle opere pubbliche alle infrastrutture: profili evolutivi". In La disciplina delle opere pubbliche, a cura di Alessandro Crosetti. Rimini: Maggioli, 2007.

De Nictolis, Rosanna. "La funzione di pianificazione urbanistica e la localizzazione e realizzazione di opere pubbliche". Cons. St., vol. 55, Fasc. 11, 2004.

De Nictolis, RosannA. "Le novità sui contratti pubblici recate dal D.L. n. 32/2019 'sblocca cantieri'". Urbanistica e. appalti., n. ${ }^{\circ}$ 4, 2019.

De SANTIS, VAlERIA. "La nuova legge della regione Toscana in materia di dibattito pubblico regionale e promozione della partecipazione". In Osservatorio costituzionale, Fasc. 0, 2013.

Della Cananea, Giacinto. "Per un nuovo assetto delle reti di servizi pubblici". Munus, n. ${ }^{\circ} 1,2011$.

Di MARTINO, AlesSANDRO. "Il dibattito pubblico per la realizzazione di grandi infrastrutture: quale ruolo per la partecipazione democratica?". Nuove aut., n. ${ }^{\circ} 3,2017$.

DiAnA, IACOPO LuCA. "La recente disciplina in tema di 'dibattito pubblicò' sull'aggiudicazione di appalti e concessioni pubbliche". Riv. giur. sarda, n. ${ }^{\circ}$, pt. II, 2016.

DipACE, RugGiero. "La logica della prevenzione nella disciplina della programmazione e progettazione di lavori pubblici". Riv. giur. edil., n. ${ }^{\circ}$ 6, 2018.

DipACE, RugGiERO. "Le reti di trasporto fra disciplina europea e nazionale per la realizzazione di una mobilità sostenibile, in Infrastrutture di trasporto e sistemi di regolazione e gestione". In Coesione, sostenibilità e finanziamenti, a cura di Giovanna Colombini, Marina D'Orsogna, Loredana Giani e Aristide Police. Napoli: Editoriale Scientifica, 2019.

DiPACE, RUGGIERO. "Procedure di approvazione dei progetti e conferenza di servizi". In Trattato sui contratti pubblici, I, a cura di Maria Alessandra Sandulli e Rosanna de Nictolis, tomo IV. Milano: Giuffrè, 2019

Draghi, MARIO. Considerazioni finali - Banca d'Italia, Assemblea Ordinaria dei Partecipanti anno 2006, Roma, 31 maggio 2007. 
DuGATO, MARCO. "Il partenariato pubblico-privato: origine dell'istituto e sua evoluzione". In La collaborazione pubblico-privato e l'ordinamento amministrativo, a cura di Franco Mastragostino. Torino: Giappichelli, 2011.

DugATO, MARCO. "La crisi del concetto di servizio pubblico locale tra apparenza e realtà". Diritto Amministrativo, n. ${ }^{\circ}$ 3, 2020.

Dugato, Marco. "Terremoto, ricostruzione e regole degli appalti". Munus, n. ${ }^{\circ}$, 2017.

FERRARA, ROSARIO. Introduzione al diritto amministrativo. Roma-Bari: Laterza, 2014.

FIDONE, GiAnFranCESCO. "Il nuovo codice dei contratti pubblici". In Commentario al codice dei contratti pubblici, a cura di Marcello Clarich. Torino: Giappichelli, 2019.

FIORENTINO, LUIGI. "I corpi tecnici delle amministrazioni: problemi attuali". Rivista trimestrale diritto pubblico, n. ${ }^{\circ} 2,2013$.

FIORITTO, Alfredo. "L'amministrazione negoziale: modelli di partenariato e problemi di applicazione". Nuove forme e nuove discipline del partenariato pubblico privato, a cura di Alfredo Fioritto. Torino: Giappichelli, 2017.

FONDAZIONE ERGO. L'Italia in-compiuta un'analisi economica delle opere pubbliche. I quaderni di approfondimento, n. ${ }^{\circ} 6,2019$, pp. 5-8

FOnderico, GiUliano. "Finanziamenti a imprese e lavoratori autonomi". Giornale di. diritto amministrativo, n. ${ }^{\circ} 5,2020$.

FRACCHIA, FABRIZIO, e LUIGI GILI. Ordinamento dell'Unione europea, mercato, risorse pubbliche e contratti della pubblica amministrazione. Profili sostanziali e processuali. Napoli: Editoriale Scientifica, 2013.

GABRIELli, EnRICO. "L'operazione economica nella teoria del contratto". Rivista. Trimestrale di diritto processuale civ., n. ${ }^{\circ} 66$ (1), 2009.

Gallia, Roberto. "La spesa per infrastrutture degli Enti locali: l'efficacia della programmazione condizionata dalle semplificazioni procedimentali". Riv. giur. mezz., Fasc. 2, 2019.

GAmbardella, FortunAto. Le regole del dialogo e la nuova disciplina dell'evidenza pubblica. Torino: Giappichelli, 2016.

Giannelli, Annalaura. "Contratti pubblici: stabilità del rapporto e interessi pubblici". Diritto economia, n. ${ }^{\circ} 1,2016$.

Giannini, Massimo Severo. Diritto pubblico dell'economia. Bologna: il Mulino, 1993. 
IMMORDINO, MARIA. "Recovery plan? Sostegno allo sviluppo ed equilibrio finanziario con il 'tesoro' dei lavori pubblici". Appalti e contrati, n. ${ }^{\circ}$ 7-8, 2020.

Libertini, Mario. Diritto della concorrenza dell'Unione europea. Milano: Giuffrè, 2014.

MaCCHiA, MARCO. "La Cassa depositi e prestiti tra i diritti e le reti di nuova generazione". Munus, n. ${ }^{\circ} 1,2014$.

MACCHIA, MARCO. "La qualificazione delle amministrazioni appaltanti". Giornale di Diritto amministrativo, Fasc. 1, 2017.

MACCHIA, MARCO. "La regolamentazione degli affidamenti da parte dei concessionari". Rivista italiana diritto pubblico comparato, n. ${ }^{\circ} 1,2018$.

MANZETTI, VANESSA. "Il 'dibattito pubblico' nel nuovo codice dei contratti". federalismi.it, n. ${ }^{\circ} 5,2018$.

Massera, Alberto. "Principi procedimentali". In Trattato sui contratti pubblici, t. I, diretto Maria Alessandra Sandulli e Rosanna de Nictolis. Milano: Giuffrè, 2019.

Massera, Alberto. Lo Stato che contratta e che si accorda. Vicende della negoziazione con le PP. AA., tra concorrenza per il mercato e collaborazione con il potere. Pisa: Edizioni Plus, 2011.

Mastrodonato, Giovanna. "Dibattito pubblico e partecipazione: le decisioni amministrative condivise nel Codice dei contratti pubblici". Munus, n. ${ }^{\circ}$ 1, 2018.

Mattarella, Bernardo Giorgio. "La centralizzazione delle committenze". Giornale di diritto amministrativo, n. ${ }^{\circ}$ 5, 2016.

MELIS, Guido. "Culture dei tecnici e amministrazione nell'Italia di ieri e di oggi". Rivista giuridica Mezzogiorno, n. ${ }^{\circ}$ 2, 2019.

MELIS, Guido. "La fuga dall'amministrazione. Ascesa e declino dei tecnici nell'amministrazione dell'Italia unita". Rivista trimestrale di diritto pubblico, n. ${ }^{\circ} 2,2013$.

MOlaSCHI, VivianA. "La democratizzazione delle decisioni 'science and technology based'. Riflessioni sul dibattito pubblico". Nuove aut., Fasc. 3, 2017.

Napolitano, GiUlio. La logica del diritto amministrativo. Bologna: il Mulino, 2020.

OCCHIEnA, Massimo. "Gli appalti pubblici e la semplificazione impossibile". Diritto dell'economia., vol. 26, n. ${ }^{\circ} 81,2013$.

OcChiena, Massimo. "Il coraggio di semplificare". Diritto dell'economia, n. ' 102, 2020. 
Otranto, Piergiuseppe. "Decisione amministrativa e digitalizzazione della p.a.". federalismi.it, n. ${ }^{\circ} 2,2018$.

PellizZer, Franco. "La programmazione e la progettazione". In Diritto dei contratti pubblici, a cura di Franco Mastragostino. Torino: Giappichelli, 2019.

PIPERATA, GiUSEPPE. "Infrastrutture e crescita economica: alla ricerca di nuovi modelli". Munus, n. ${ }^{\circ}$ 2, IV, 2013.

PIPERATA, GiUSEPPE. "Introduzione". In La collaborazione pubblico-privato e l'ordinamento amministrativo, a cura di Franco Mastragostino. Torino: Giappichelli, 2011

Pirozzoli, AnNA. "L'istituto del 'dibattito pubblico' sulle grandi opere". Rass. parl., vol. 60 , n. ${ }^{\circ} 2,2018$.

PISANESCHI, ANDREA. Dallo Stato imprenditore allo Stato regolatore e ritorno? Torino: Giappichelli, 2009.

Protto Mariano e Luca Mazzeo. "Project financing e infrastrutture strategiche: aspetti di criticità dell'attuale sistema e loro (possibile) superamento alla luce della direttiva 2014/23/Ue". In AA. VV., Finanza di progetto e partenariato pubblico privato, 2015.

RICCHI, MASSIMO. "Negoauction, discrezionalità, dialogo competitivo e il nuovo promotore". In Le gare pubbliche: il futuro di un modello, a cura di Gian Domenico Comporti. Napoli: Editoriale Scientifica, 2011.

ROBERTS DAVID. Victorian Origins of the British Welfare State. New Heaven: Yale University Press, 1960.

SALTARI, LORENZO. "Che resta delle strutture tecniche nell'amministrazione pubblica italiana?". Rivista trimestrale di diritto pubblico, n. ${ }^{\circ}$ 1, 2019.

Salvemini, Maria Teresa. "La posizione di Cassa depositi e prestiti". Rivista giuridica mezzogiorno, n. ${ }^{\circ} 1,2015$.

SANDULli, AlDO. "Il codice dei contratti pubblici tra resistenze nazionali e spinte europee". Munus, n. ${ }^{\circ}$ 3, 2019.

SANDUlli, Maria Alessandra. "Principi e regole dell'azione amministrativa': riflessioni sul rapporto tra diritto scritto e realtà giurisprudenziale". federalismi. it, n. ${ }^{\circ} 23,2017$.

Sandulli, Maria Alessandra. "Ancora sui rischi dell'incertezza delle regole (sostanziali e processuali) e dei ruoli dei poteri pubblici. Postilla a 'Principi e 
regole dell'azione amministrativa. Riflessioni sul rapporto tra diritto scritto e realtà giurisprudenziale'". federalismi.it, n. ${ }^{\circ}$ 11, 2018.

Sbrescia, VinCEnZO M., "Alberto Beneduce e le trasformazioni del modello di interventi pubblico in economia: la vicenda dello Stato imprenditore ed il ruolo dell'Iri a quindici anni dalla sua messa in liquidazione". Riv. giur. mezz., v. 1, 2015.

Sciullo, Girolamo. "Concertazione istituzionale e localizzazione delle grandi opere". Giorn. dir. amm., vol. 10, 2004.

SCOCA, FRANCO GAETANO. "Il coordinamento e la comparazione degli interessi nel procedimento amministrativo". In Convivenza nella libertà. Scritti in onore di Giuseppe Abbamonte, II. Napoli: Jovene, 1999.

SCREPANTI, SUSANNA. "I tempi delle opere pubbliche e la relatività del tempo nel diritto amministrativo". Riv. trim. dir. pubbl., n. ${ }^{\circ}$ 4, 2019.

SCREPANTI, SUSANNA. "Le politiche infrastrutturali". In La nuova costituzione economica, a cura di Sabino Cassese. Bari-Roma: Laterza, 2021.

SEBASTIANI, MARIO. "Le infrastrutture di trasporto: programmazione, concorrenza, interesse pubblico". In I nodi delle reti. Infrastrutture, mercato e interesse pubblico, a cura di Paola Manacorda. Firenze: Passigli Editori, 2010.

Serra, Michele. "Grandi opere. Gli ostinati del bluff", in La Repubblica, 8 de agosto de 2006.

Servizio Studi Della CAMERA Dei DeputATI. Infrastrutture strategiche e prioritarie. Programmazione e realizzazione. Rapporto 2020 in collaborazione con l'Autorità nazionale anticorruzione, Roma, 2020.

SGueO, GianluCA. "L'amministrazione digitale". Giornale di dritto amministrativo, n. ${ }^{\circ} 1$, 2016.

TORCHIA, LUISA. "Tendenze recenti della semplificazione amministrativa". Diritto amministrativo, $\mathrm{n}^{\circ}{ }^{\mathrm{3}}$-4, 1998.

TRAVI, AlDO. "Il partenariato pubblico-privato: i confini incerti di una categoria". In Negoziazioni pubbliche, a cura di Maurizio Cafagno, Alessandro Botto, Gianfrancesco Fidone e Gabriele Bottino. Milano: Giuffrè, 2013.

TRIMARCHI BANFI, FRANCESCA. "I rapporti contrattuali della pubblica amministrazione. Diritto pubblico", 1998.

TRIMARCHI BANFI, FRANCESCA. "Il "principio" di concorrenza: proprietà e fondamento". Diritto amministrativo, n. ${ }^{\circ}$ 1-2. 2013. 
Tropea, Giuseppe e AnNalaura Giannelli. "L'emergenza pandemica e i contratti pubblici: una 'semplificazione' in chiaroscuro, tra misure temporanee e prospettive di riforma strutturale". Munus, Fasc. 2, 2020.

VAlaguzZa, SARA E EduARdo PARISI. "Ricerca sull'identità giuridica del partenariato pubblico-privato". Munus, n. ${ }^{\circ} 1,2020$.

VALVO, AnNA LuCia. "'Soft law' e (in)certezza del diritto con particolare riguardo all'ordinamento dell'Unione europea". Riv. coop. giur. internaz., n. ${ }^{\circ}$ 64, 2020.

VinTI, STEFAnO. "L'eterogenesi dei fini tradisce lo scopo ultimo e l'oggetto stesso della disciplina sui contratti pubblici". federalismi.it, n. ${ }^{\circ}$ 12, 2020.

VISCO, IGNAZIO. "Investimenti pubblici per lo sviluppo dell'economia". In Sviluppo economico, vincoli finanziari e qualità dei servizi: strumenti e garanzie. Atti del LXIV Convegno di studi di scienza dell'amministrazione promosso da Provincia di Lecco, Corte dei conti, Varenna-Villa Monastero, 20-21-22 settembre 2018, Milano: Giuffrè, 2019.

World ECONOmic Forum. The Global Competitiveness Report 2019. Swizerland, 2019.

ZunARELl, SteFAnO. "Un nuovo strumento di prevenzione dei conflitti nella realizzazione delle grandi opere portuali: il dibattito pubblico". Diritto marittimo, Fasc. 3, 2018. 